\title{
Alix and Syntenin-1 direct amyloid precursor protein trafficking into extracellular vesicles
}

\author{
Allaura S. Cone ${ }^{1 \dagger}$, Stephanie N. Hurwitz ${ }^{1 \dagger}$, Gloria S. Lee ${ }^{1}$, Xuegang Yuan², Yi Zhou', Yan Li and \\ David G. Meckes $\mathrm{Jr}^{1 *}$ (D)
}

\begin{abstract}
Background: Endosomal trafficking and amyloidogenic cleavage of amyloid precursor protein (APP) is believed to play a role in the neurodegeneration observed in Alzheimer's disease (AD). Recent evidence has suggested that packaging and secretion of APP and its amyloidogenic cleaved products into small extracellular vesicles (EVs) may facilitate uptake of these neurotoxic factors during disease progression. However, the molecular mechanisms underlying trafficking of APP into EVs are poorly understood.

Results: In this study, the mechanism and impact of APP trafficking into extracellular vesicles (EVs) were assessed by a series of inducible gene knockdowns. We demonstrate that vesicle-associated proteins Alix and Syntenin-1 are essential for proper subcellular localization and efficient EV secretion of APP via an endosomal sorting complexes required for transport (ESCRT)-independent pathway. The neurotoxic C-terminal fragment (CTF $\beta$ ) of APP is similarly secreted in association with small vesicles. These mechanisms are conserved in terminally differentiated neuron-like cells. Furthermore, knockdown of Alix and Syntenin-1 alters the subcellular localization of APP, sequestering the precursor protein to endoplasmic reticulum and endolysosomal compartments, respectively. Finally, transfer of small EVs containing mutant APP confers an increase in reactive oxygen species production and neurotoxicity to human induced pluripotent stem cell-derived cortical neurons and naïve primary neurons, an effect that is ameliorated by Alix and Syntenin-1 depletion.
\end{abstract}

Conclusions: Altogether these findings elucidate a novel mechanism for understanding the intracellular trafficking of APP and CTF $\beta$ into secreted extracellular vesicles, and the resultant potential impact on neurotoxicity in the context of Alzheimer's disease amyloidopathy.

Keywords: Extracellular vesicle, Exosomes, Alzheimer dementia, Neurodegeneration, Multivesicular bodies, Protein trafficking

\footnotetext{
* Correspondence: david.meckes@med.fsu.edu

${ }^{\dagger}$ Allaura S. Cone and Stephanie N. Hurwitz should be considered joint first author

${ }^{1}$ Department of Biomedical Sciences, Florida State University College of Medicine, 1115 West Call Street, Tallahassee, FL 32306-4300, USA

Full list of author information is available at the end of the article
}

(c) The Author(s). 2020 Open Access This article is licensed under a Creative Commons Attribution 4.0 International License, which permits use, sharing, adaptation, distribution and reproduction in any medium or format, as long as you give appropriate credit to the original author(s) and the source, provide a link to the Creative Commons licence, and indicate if changes were made. The images or other third party material in this article are included in the article's Creative Commons licence, unless indicated otherwise in a credit line to the material. If material is not included in the article's Creative Commons licence and your intended use is not permitted by statutory regulation or exceeds the permitted use, you will need to obtain permission directly from the copyright holder. To view a copy of this licence, visit http://creativecommons.org/licenses/by/4.0/ The Creative Commons Public Domain Dedication waiver (http://creativecommons.org/publicdomain/zero/1.0/) applies to the data made available in this article, unless otherwise stated in a credit line to the data. 


\section{Background}

Alzheimer's disease (AD) remains the major cause of dementia among the elderly, yet despite significant attention to the rising burden of disease, little is certain with regards to the mechanism of $\mathrm{AD}$ development and progression. Despite questions surrounding the mechanisms of neurodegeneration in $\mathrm{AD}$, abundant research has pointed to the involvement of amyloid beta $(A \beta)$ in producing neurotoxicity seen in the course of the developing dementia. Early in vitro work has demonstrated that $A \beta$ fibrils, especially those formed by aggregated $\mathrm{A} \beta 42$ monomers, are highly toxic to neuronal cells in culture $[1,2]$. A number of pathogenic mutations in amyloid precursor protein (APP) that lead to increased $\mathrm{A} \beta$ production have been identified, including the Swedish mutation $(\mathrm{K} 670 \mathrm{~N} / \mathrm{M} 671 \mathrm{~L})$ at the amino terminus of the $\mathrm{A} \beta$ region and the Indiana mutation (V717F), among others [3-5]. Subsequent in vivo transgenic mouse lines have been developed which overexpress amyloidogenic variants of human APP [6]. These mutations lead to increased amyloidogenic cleavage of APP, significant amyloid deposition and inflammatory response by 4 to 6 months, and detectable impairment on spatial memory testing. The Alzheimer's-like phenotype of APP mutant mice has generated much interest in understanding the physiological role of APP, in addition to its processing and intracellular trafficking.

The APP gene is located on human chromosome 21q21.3 and gives rise to three major isoforms, with APP695 (a 695 amino acid protein) most predominately expressed in neurons [7, 8]. The full-length protein is a single pass transmembrane protein with an intracellular C-terminus, and is synthesized in the endoplasmic reticulum before transportation through the Golgi and trans-Golgi network into secretory vesicles to the plasma membrane. On the cell surface, APP can be proteolytically cleaved by $\alpha$ secretase then $\gamma$-secretase to generate a soluble ectodomain product (sAPP $\alpha)$, which may be neuroprotective [9-11]. Unprocessed APP is internalized into the endosomal compartment, which contains $\beta$ secretase enzymes that cleave APP to produce sAPP $\beta$ and $\mathrm{B}$-secretase derived $\mathrm{C}$-terminal fragment of APP $(\mathrm{CTF} \beta)$. Next, $\gamma$-secretase cleaves $\operatorname{CTF} \beta$ to produce $\mathrm{CTF} \gamma$ and $\mathrm{A} \beta[12,13]$. Recent research identifying new secretases such as $\delta$-secretase, $\eta$-secretase, and meprin- $\beta$ have added to the intricacy of APP processing [14]. These downstream derivatives have been a topic of recent research, particularly with regards to elucidating the role these different metabolites play in both $\mathrm{AD}$ and healthy cells. Interestingly, evidence suggests that trafficking of APP into lipid rafts and localization to acidic endosomes, where the $\beta$-secretase BACE is present, is necessary for amyloidogenic processing [15-19].

More recent studies have demonstrated that APP and its neurotoxic $\beta$-secretase derived catabolites, CTF $\beta$ and $A \beta$, are trafficked into secreted extracellular vesicles (EVs), including endosomal-derived exosomes [20-23]. It is worth noting that CTF $\beta$ has also been found to lead to inflammation and synaptic dysfunction [24] and may accumulate before $A \beta$ peptides in the brains of $\mathrm{AD}$ mice and human patients [25, 26]. It has been suggested that CTF $\beta$ is particularly important in the preliminary stages of $\mathrm{AD}$ progression, and accumulation may lead to early lesions in the hippocampus [25]. With regards to EV secretion, intercellular transport of APP or the $\beta$-secretase derived catabolites likely has a harmful effect on naïve neurons [21], while inhibition of EV release may result in decreased amyloid plaque load present in AD mouse models [27]. Decreased release of small EVs has been found to augment oligomerized CTF $\beta$ accumulation in the endolysosomal compartments of cells [28]. This intracellular aggregation could lead to endosomal dysfunction, which is an early feature of AD [24].

Despite the significance of EV-associated secretion of APP, how APP is packaged into these vesicles is largely unknown. In general, several molecular pathways of small EV formation and cargo packaging are regarded, including processes involving endosomal sorting complexes required for transport (ESCRT) complexes [2931]. The ESCRT pathway consists of four distinct protein complexes (ESCRT -0,-I,-II, and -III) in addition to several ESCRT-associated proteins (Alix, Vps4a, and Vta1) [32, 33]. Briefly, the ESCRT-0 complex is comprised of Hepatocyte growth factor-regulated tyrosine kinase substrate (Hrs) and Signal transducing adaptor molecule (Stam) proteins which bind and sequester ubiquitinated cargo for delivery to multivesicular bodies (MVBs) [31]. Hrs is responsible for recruitment of the ESCRT-I protein Tsg101, and the ESCRT-II complex subsequently assembles to guide MVB biogenesis and membrane budding, forming intraluminal vesicles later secreted as exosomes. ESCRT-associated protein Alix aids in drafting the ESCRT-III complex to the endosomal membrane to guide membrane scission and vesicle formation in MVBs [31]. Additional evidence suggests Alix also interacts with syndecans and an adaptor protein Syntenin-1, which facilitate vesicle protein trafficking through binding of syndecan, a type of heparan sulphate proteoglycan, with numerous ligands in an ESCRT-independent manner [34, 35]. In other scenarios, vesicle production and cargo packaging may instead be dependent on tetraspanin-mediated biogenesis or ceramide-driven membrane budding [36-40]. 
Here, we corroborate previous research [20-23] showing enrichment of wild-type and Swedish mutant amyloid precursor protein (APP ${ }^{\mathrm{WT}}$ and $\mathrm{APP}^{\text {swe }}$ ) and its CTF $\beta$ metabolite into small EVs from HEK293 cells, in addition to differentiated SH-SY5Y neuronal cells. Through gene knockdown (KD) analyses, we further demonstrate that secretion of these AD-associated proteins is dependent upon an Alix- and Syntenin-1 mediated mechanism of vesicle cargo sorting. Cellular localization of APP is largely disrupted following Alix and Syntenin-1 KD, suggesting the importance of the previously recognized Alix-Syntenin-1 pathway in trafficking the amyloid precursor protein within cells. Finally, we reveal that Alix and Syntenin-1 depletion ameliorates the reactive oxygen species production and neurotoxicity observed following transfer of APP- and
CTF $\beta$ - containing EVs onto naïve neuronal cells. Altogether these findings elucidate a novel mechanism for APP sorting, processing, and secretion from cells, which likely has downstream consequences in the context of AD progression.

\section{Results}

Mutant amyloid precursor protein mutant is secreted into small EVs

Amyloid precursor protein harboring the Swedish mutation has previously been demonstrated to be secreted into EVs, and transmitted intercellularly [21]. Here, we demonstrate the co-enrichment of $\mathrm{APP}^{\text {swe }}$ and other small EV proteins in vesicles following ultracentrifugation at 100,000 g (Fig. 1a). Enriched EVs were devoid of Calnexin, an intracellular endoplasmic reticulum

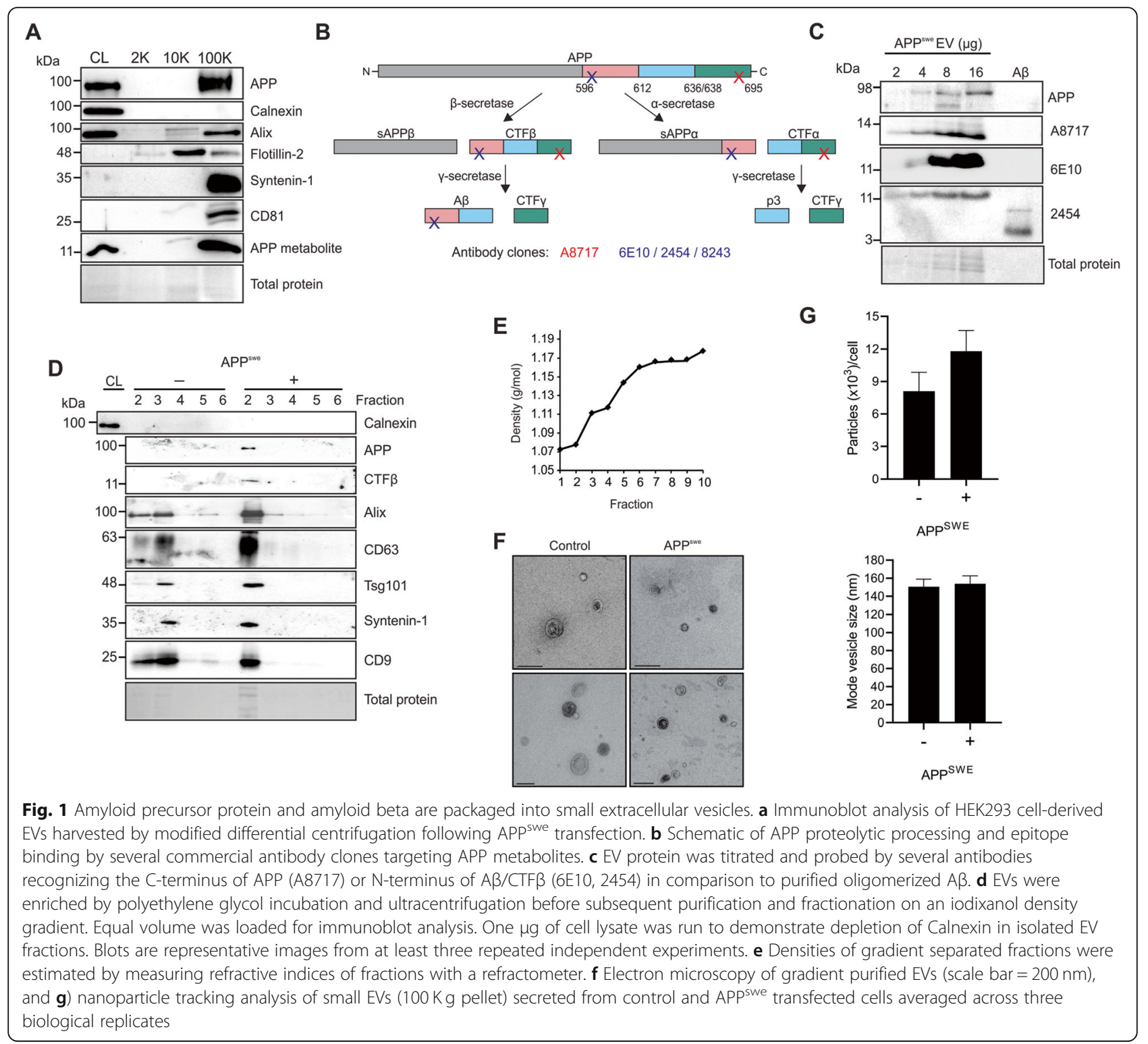


protein. Interestingly, APP and its $\beta$-secretase cleaved metabolite were not present in large vesicles pelleted at $2000 \mathrm{~g}$, and only trace amounts of APP metabolites were isolated in medium-sized Flotillin-2 enriched vesicles pelleted at $10,000 \mathrm{~g}$.

It is well documented that antibodies targeting the secretase-cleaved products may display cross-reaction, particularly between $A \beta, C T F \alpha$, and CTF $\beta$. To distinguish the identity of the predominant APP metabolite seen in EVs, several antibodies targeting either the Cterminus of APP, or the $\mathrm{N}$-terminus of $\mathrm{A} \beta$ were used (Fig. 1b). Titrated EV protein showed increasing levels of a metabolite between 11 and 14 kilodaltons $(\mathrm{kDa})$ that was detectable with all antibodies used (Fig. 1c). Comparison to purified $A \beta$ oligomer detected between 4 and $5 \mathrm{kDa}$ revealed CTFs to be the most prevalent metabolite in EVs rather than $A \beta$. Furthermore, primary probing with antibody clone $6 \mathrm{E} 10$ which binds the N-terminal of $\mathrm{A} \beta$ or CTF $\beta$ detected a band of similar molecular mass, indicating that the identify of this metabolite was most likely consistent with CTF $\beta$ rather than CTF $\alpha$ (Fig. 1b and $\mathrm{c}$ ). It is possible that other cleaved products were present in EVs at lower levels. The remainder of immunodetection of CTF $\beta$ was performed using antibody clones 2454 and 8243.

Small EVs were further purified on an iodixanol density gradient, where APP was found to co-migrate with proteins associated with small EVs, including Tsg101, CD63, CD9, Syntenin-1, and Alix, to fractions previously demonstrated to be consistent with the density of exosomal-enriched isolates [40-43] (Fig. 1d-e). Vesicles enriched from cells expressing the mutant APP demonstrated no significant difference in morphology, size, or quantity compared to EVs from normal control cells by electron microscopy (Fig. 1f) and nanoparticle tracking (Fig. 1g).

\section{Alix and Syntenin-1 guide vesicle packaging of APP}

Various ESCRT- dependent or -independent pathways are involved in trafficking and secretion of EV cargo. In this study, we utilized a series of gene knockdowns to elucidate the major mechanism of APP sorting into small EVs. Efficient knockdowns of ESCRT pathway proteins Hrs and Tsg101 were achieved, in addition to knockdowns of the ESCRT-associated protein Alix, tetraspanin protein CD63, and Syntenin-1 (Fig. 2a). Strikingly, in cells expressing APP ${ }^{\text {swe }}$, the mutant precursor protein was packaged into EVs despite considerable loss of Hrs, Tsg101, and CD63 protein expression (Fig. 2b). However, efficient APP packaging into small EVs appeared to be dependent upon the presence of Alix and Syntenin-1 (Fig. 2b and d). Endogenous APP secretion was likewise diminished with Alix and Syntenin-1 knockdown (Fig. 2c). Interestingly, knockdown of Alix decreased the amount of Syntenin-1 secreted into vesicles, and a similar effect was seen in Alix release following Syntenin-1 knockdown (Fig. 2b), supporting a common pathway of EV sorting including these two proteins. We note that the relatively decreased quantity of APP secreted into EVs following CD63 knockdown may have been primarily due to increased cellular levels of APP, the mechanism of which is not understood, but could be due to intracellular trafficking or degradation variation in the setting of CD63 protein depletion.

As expected, untransfected cells expressing endogenous APP demonstrated very little secretion of APP or CTF $\beta$ into EVs (Fig. 2d). However, higher levels of CTF $\beta$ derived from $\mathrm{APP}^{\text {swe }}$ mutant cells were secreted in association with vesicles, and similarly dependent on Alix and Syntenin-1 for packaging (Fig. 2d and f). Interestingly, decreased secretion of APP and CTF $\beta$ was not a factor of overall diminished EV production. In fact, depletion of Alix and Syntenin-1 appeared to increase total vesicle production even slightly (Fig. $2 \mathrm{~g}$ ), though the source of these EVs remains unknown. No significant difference in vesicle size was seen following Alix or Syntenin-1 knockdown (Fig. 2h).

It has been reported that overexpression of ESCRT-II proteins may rescue depletions of earlier ESCRT components [30,31]. Therefore, it was considered possible that APP release was rescued by a downstream ESCRT compensatory pathway, rather than packaged into vesicles entirely independent of early ESCRT proteins. To address this question, we introduced a GFP-tagged dominant negative mutant of Vps4a (Vps4a E228Q) [44], an AAA ATPase that plays an essential role in ESCRT complex disassembly, membrane deformation, and fission events crucial for the final stages of intraluminal vesicle formation (Fig. 3a). Vesicle secretion of early ESCRT proteins Hrs and Tsg101 appeared mildly increased following overexpression of wild-type Vps4a (Fig. 3b, d, e). However, in the presence of the dominant negative Vps4a protein, Hrs and Tsg101 secretion was reduced or returned to baseline. Notably, a decrease in APP or CTF $\beta$ vesicle packaging was not observed in cells expressing the dominant negative Vps4a protein (Fig. 3bc), affirming the suspected ESCRT-independent sorting pathway.

To further determine whether the Alix and Syntenin-1 reliant sorting of APP was specific to the mutant protein, human wild-type APP was introduced into cells carrying the genetic knockdowns (Fig. 4a). Of note, high levels of transfected wild-type APP were sufficient to produce detectable levels of the catabolite CTF $\beta$ in cells, although CTF $\beta$ derived from APP ${ }^{\mathrm{WT}}$ was not efficiently secreted into EVs. In these experiments, secretion of wild-type APP was similarly diminished following Alix, Syntenin-1 knockdown (Fig. 4b-c). Again, CD63 


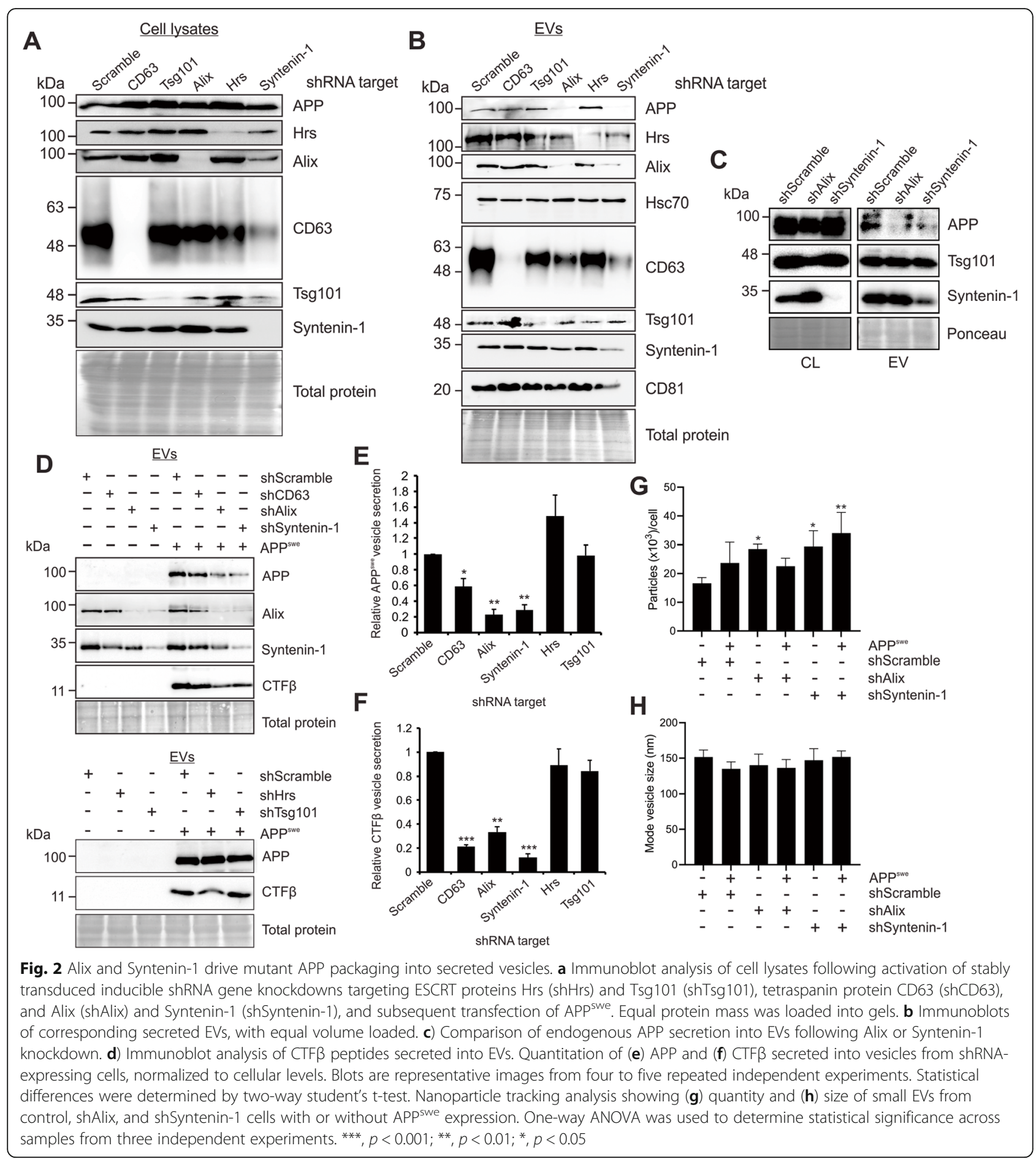

knockdown slightly reduced vesicle APP ${ }^{\mathrm{WT}}$ secretion, while a mild increase in cellular levels of APP were noted. Interestingly, knockdown of earlier ESCRT proteins Hrs and Tsg101 also reduced APP ${ }^{\mathrm{WT}}$ vesicle packaging. Again, inhibition of Vps4a using the dominant negative protein Vps4a E228Q demonstrated no significant impact on APP or A $\beta$ packaging (Fig. 4d-e). These findings suggest APP is normally sorted into EVs through both ESCRT and Alix-Syntenin-1 pathways, but the mutant protein may rely more heavily on the AlixSyntenin-1 pathway or can escape earlier ESCRT protein knockdowns.

Elucidation of the mechanisms surrounding vesicular trafficking of APP and its catabolites is perhaps most relevant in the context of neuronal EV secretion. To examine whether this apparent mechanism of APP 


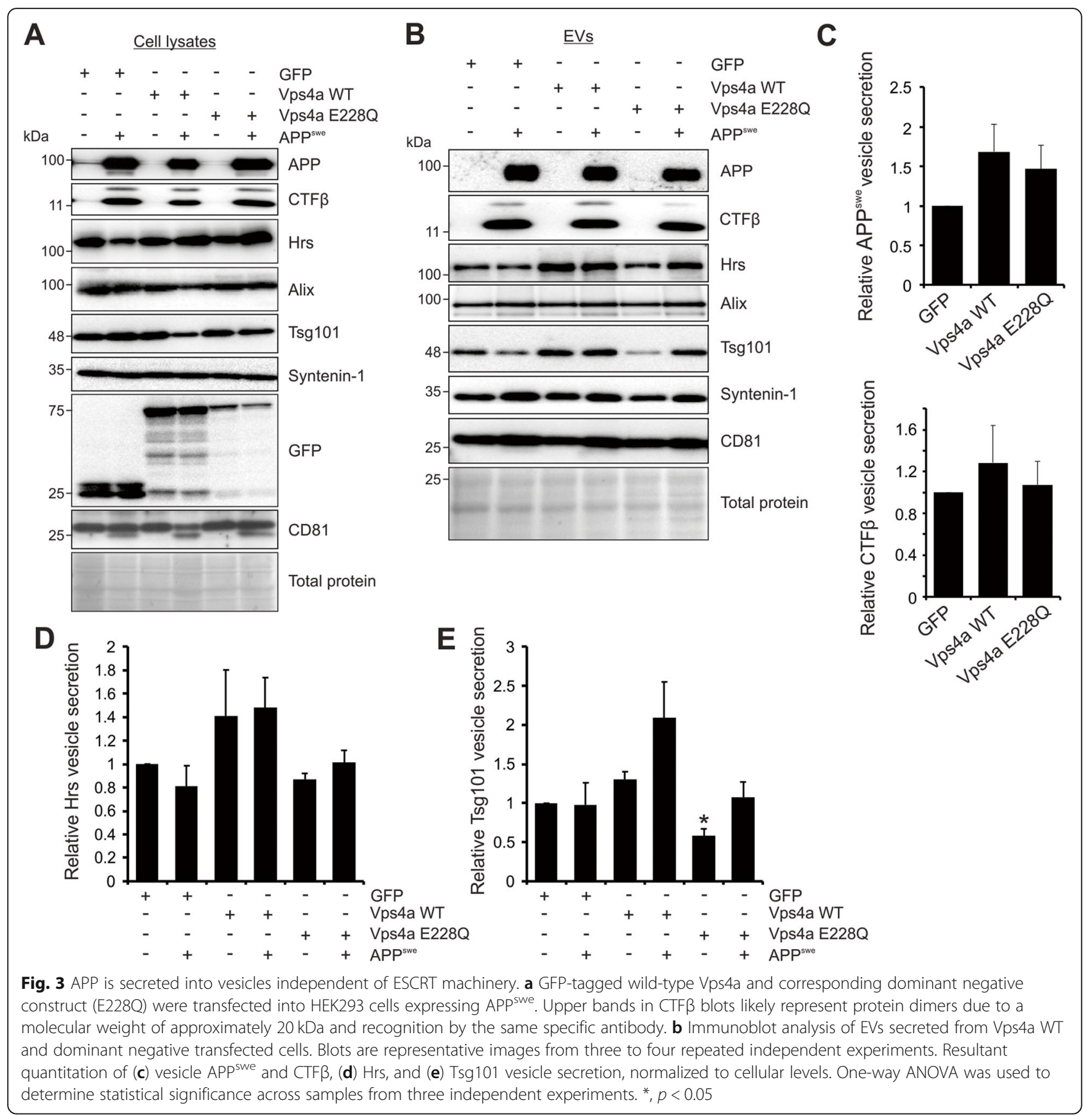

sorting was conserved, human neuroblastoma (SHSY5Y) cells containing inducible Alix shRNA were differentiated into mature neurons. Decreased vesicle secretion of both Alix and Syntenin-1 was seen following Alix knockdown (Fig. 5a). Similar to the results observed in HEK293 cells, APP and CTF $\beta$ secretion was also significantly reduced in these cells, suggesting a comparable mechanism of APP trafficking and vesicle secretion (Fig. 5b). Interestingly, intracellular CTF $\beta$ levels appeared increased following Alix depletion. It is possible that impaired trafficking of the precursor protein leads to differential amyloidogenic processing, or perhaps degradation mechanisms are unable to compensate for the impaired secretion. Finally, evaluation of endogenous APP secretion demonstrated similar dependency on Alix in SH-SY5Y cells (Fig. 5c).

\section{Intracellular localization of APP is dependent upon Alix and Syntenin-1}

To determine whether intracellular localization of the $\mathrm{APP}^{\text {swe }}$ protein was altered with Alix and Syntenin-1 knockdowns, cellular APP immunostaining was 


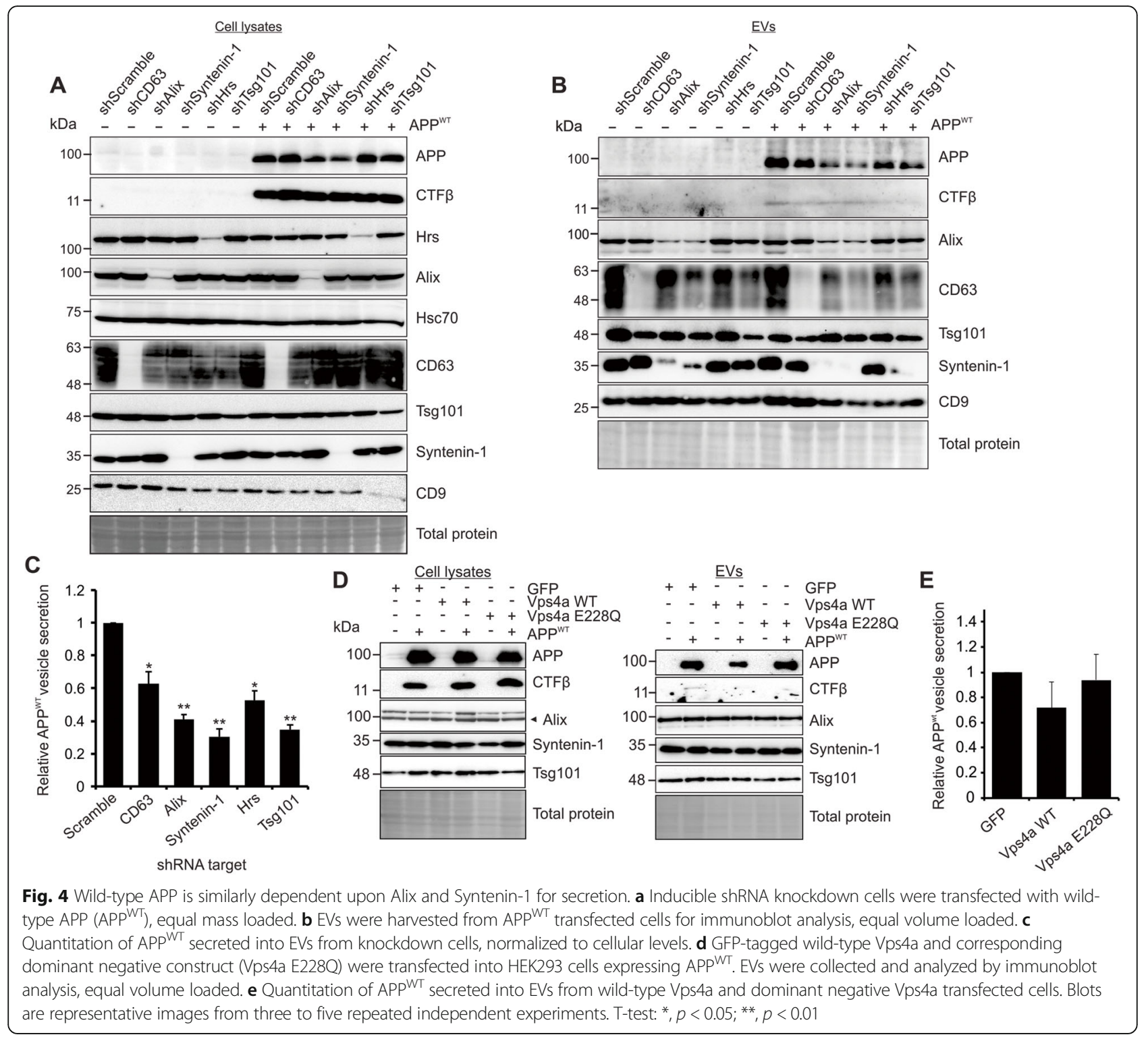

performed and imaged by confocal microscopy (Fig. 6a). APP ${ }^{\text {swe }}$ localized to several compartments in control cells, with prominent localization to the plasma membrane and perinuclear region. In contrast, Alix knockdown produced a distinct phenotype where APP was concentrated much more in the perinuclear area, while Syntenin-1 knockdown cells demonstrated a punctate localization of APP. To further characterize subcellular compartment localization of the amyloid precursor protein, a GFP-tagged $\mathrm{APP}^{\text {swe }}$ construct was expressed in control cells or those containing doxycycline-inducible Alix or Syntenin-1 shRNA. In control cells, APP localized to several subcellular compartments including 1,2-Dihexadecanoylsn-Glycero-3-Phosphoethanolamine (DHPE)-positive endosomes, lysosomes, the endoplasmic reticulum
(ER), and Golgi network (Fig. 6b-c, 7a-b). These findings are consistent with the known trafficking route of the protein. In contrast, following Alix knockdown, APP primarily localized to the ER and Golgi (Fig. 7ab), while endolyososomal localization of the protein was unchanged (Fig. 6b-c), and plasma membrane localization was attenuated. Finally, in Syntenin-1 shRNA expressing cells, APP was enriched in the lysosomal compartments (Fig. 6c), and decreased in DHPE-positive endosomes (Fig. 6b). No significant change in MDC-positive autophagic vacuole localization of APP was seen across cell groups. These alterations noted in APP trafficking within cells likely explain the reductions in vesicle secretion of the precursor protein and CTF $\beta$ in the absence of Alix and Syntenin-1. 

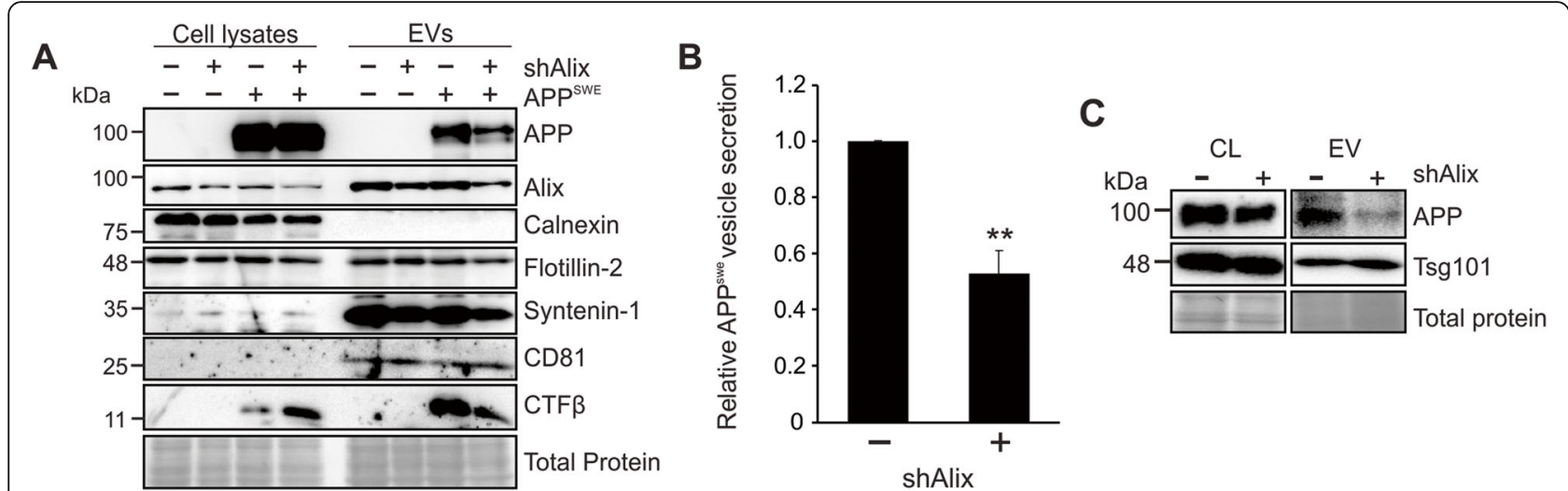

Fig. 5 Alix-dependent APP trafficking is conserved in neuron-like cells. a SH-SY5Y neuroblastoma cells stably transduced with inducible shRNA targeting Alix were terminally differentiated into neuron-like cells, then transfected with APP ${ }^{\text {swe }}$. Cell lysates and cell-derived EVs were harvested for immunoblot analysis. b Quantitation of APPswe secretion into vesicles. c Representative immunoblots of cellular levels and vesicular secretion of endogenous SH-SY5Y APP. Blots are representative images from two to three repeated independent experiments. T-test: ${ }^{*}, p<0.01$

\section{Knockdown of Alix and Syntenin-1 ameliorates the neurotoxic effect of APP}

Finally, the neurotoxic effects of APP-containing EVs were compared to those of EVs following Alix and Syntenin-1 knockdown. Primary neuronal cells were prepared from the cerebral cortex of day 0 mice and cultured in vitro. Cells were stained with anti-Map2 to demonstrate neuronal identity (Fig. 8a). Following treatment with puromycin, purified oligomerized amyloid beta, or cell-derived EVs, differentiated neuronal cells were stained with Annexin V (green) and propidium iodide (PI; red) to assess levels of apoptosis and cellular necrosis, respectively (Fig. 8b). EVs secreted from control cells conferred relatively low levels of neurotoxicity, while transfer of $\mathrm{APP}^{\text {swe }}$-containing vesicles resulted in cell death levels comparable to purified $A \beta$ peptide and puromycin-treated cells (Fig. 8b-c). This EV-mediated neurotoxic effect was significantly diminished following Alix or Syntenin-1 knockdown. Interestingly, necrotic cell death appeared to predominate in the setting of purified amyloid beta peptide, while EVs mediated mostly apoptotic cell death.

Previous research has focused on the contribution of increased reactive oxygen species (ROS) formed within cells during the progression of $\mathrm{AD}$ [45]. To assess the impact of EV treatment on ROS formation, human induced pluripotent stem (iPSK3) cells were differentiated into cortical neurons through spheroid formation in suspension. A flow cytometry-based assay was used to measure ROS production in spheroid cultures following treatment with purified $A \beta$ peptide or EVs derived from control HEK293 cells, cells expressing APP ${ }^{\mathrm{WT}}$, or cells expressing $\mathrm{APP}^{\text {swe }}$ in addition to shRNA targeting Alix or Syntenin-1. EVs containing APP ${ }^{\text {swe }}$ secreted from control cells conferred high levels of ROS, near comparable to spheroids treated with amyloid beta peptide
(Fig. 9a-b). Depletion of Alix and Syntenin-1 significantly mitigated this effect on ROS production. These findings expectedly account for, at least in part, the decrease in overall neurotoxicity seen following the transfer of similar EVs, likely a factor of decreased mutant APP and CTF $\beta$, and perhaps low levels of $A \beta$, in vesicles following Alix or Syntnein-1 knockdown.

It is believed that the neurotoxicity elicited by $A \beta$ is mediated by extracellular binding of the peptide to putative $A \beta$-binding receptors on the neuronal cell surface [46]. To determine whether APP and CTF $\beta$ are present on the surface of isolated EVs and thereby able to mediate an analogous effect, EVs were treated with trypsin to cleave external protein epitopes. Following trypsin treatment and ultracentrifugation wash, immunoblot analysis was performed on lysed EV protein (Fig. 9c). Levels of detectable APP and CTF $\beta$ were decreased in addition to transmembrane protein $\mathrm{CD} 71$. In contrast, intravesicular levels of Syntenin-1 remained unchanged. Therefore, it is likely that the cleaved APP products remain predominately associated with the external surface of vesicles, mediating their neurotoxic effects.

\section{Discussion}

The contribution of amyloid protein deposition to pathologic neurodegeneration has been a traditional and durable hypothesis with regards to the progression of Alzheimer's disease. Accruing evidence has pointed to the roles of extracellular vesicles in the secretion and uptake of misfolded proteins, including amyloid, synuclein, and prion proteins $[20,21,23$, $47-52]$. It is noted that only a small proportion of secreted $A \beta$ is likely associated with EVs $[23,53]$, as similarly concluded in this study. Given the transmembrane nature of the precursor protein and its $\mathrm{C}$ - 

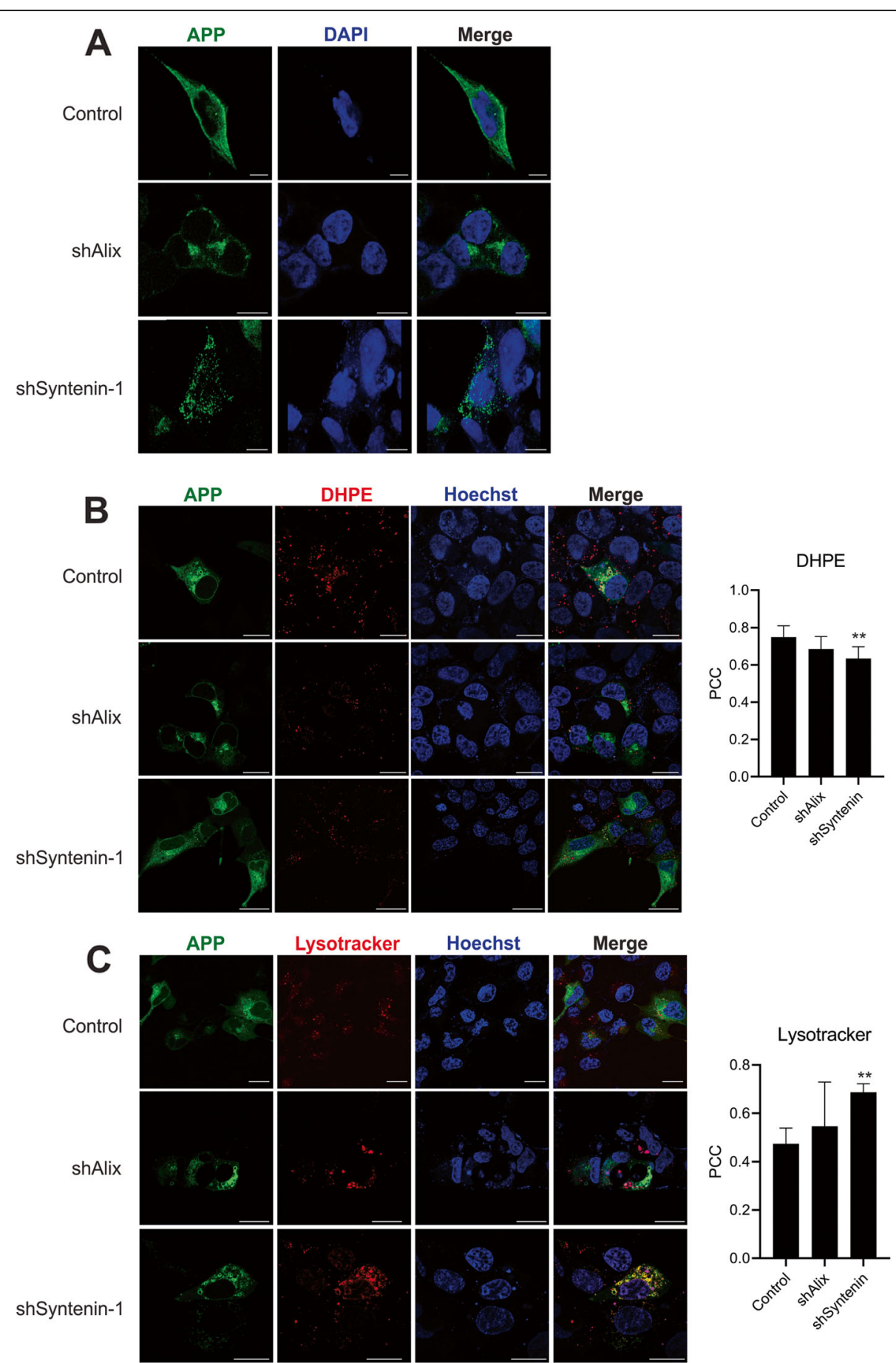

Fig. 6 APP endolysosomal localization is altered in the absence of Alix and Syntenin-1. a APP immunofluorescent staining was performed on control HEK293 cells expressing APp swe or following induction of Alix (shAlix) or Syntenin-1 (shSyntenin-1) shRNA knockdown. To assess subcellular compartment localization of APPswe in the absence of Alix and Syntenin, GFP-tagged APP ${ }^{\text {swe }}$ was introduced into cells. Cells were stained with (b) DHPE or (c) Lysotracker before live-cell imaging on a Zeiss LSM 880 confocal microscope. Images are representative across at least three independent experiments. Scale bar $=20 \mu \mathrm{m}$. Pearson's Correlation Coefficient (PCC) calculated from $\geq 10$ cells. One-way ANOVA: ** $p<0.01$. ${ }^{*}, p<0.05$

terminal fragment, the enrichment of circulating APP and CTF $\beta$ in EV membranes is expected, though not demonstrated in this study.
The net effect of EV secretion of amyloid precursor protein and its neurotoxic catabolites is not entirely clear, however. Several studies have proposed that $A \beta$ - 


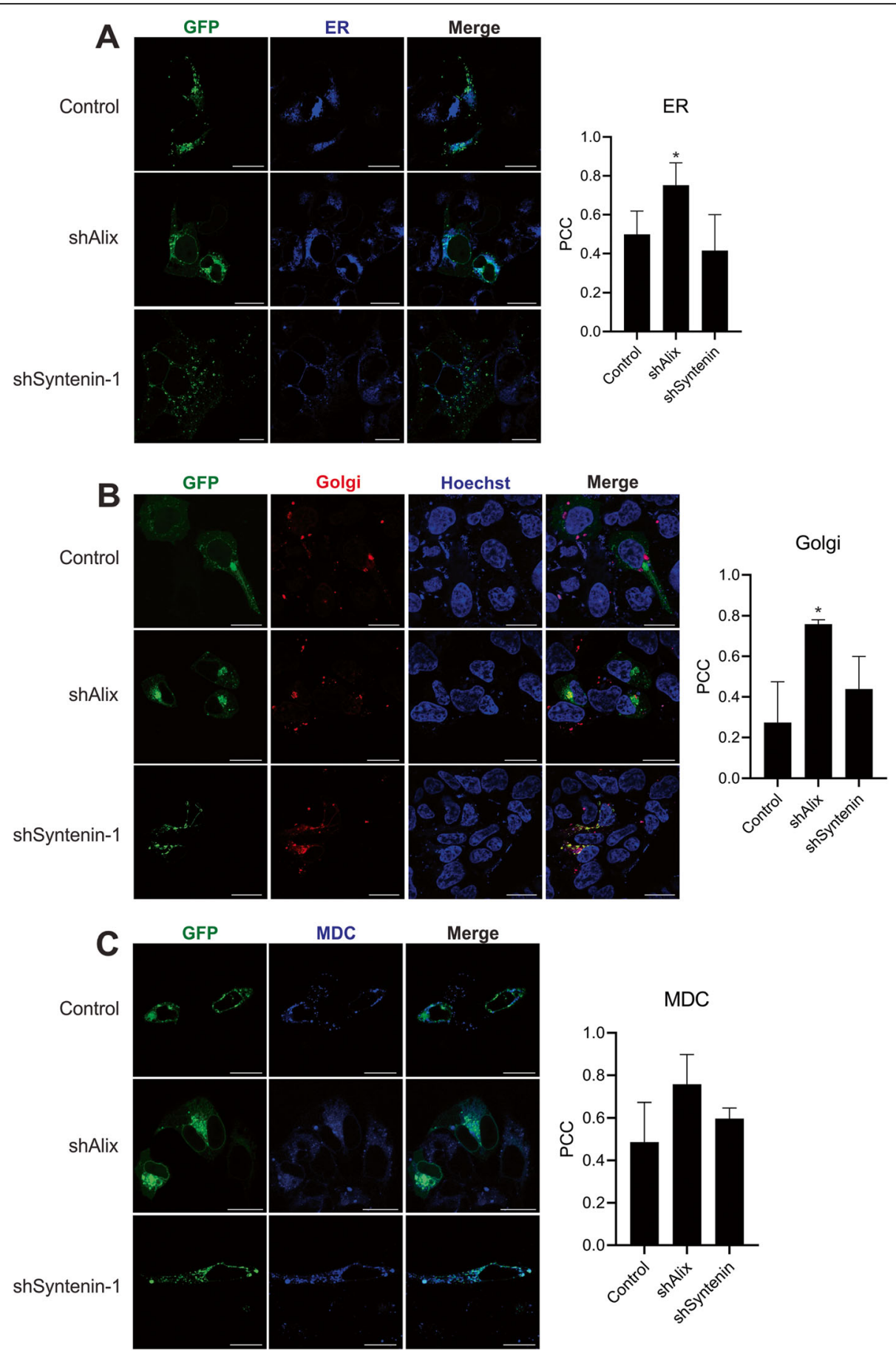

Fig. 7 APP endoplasmic reticulum (ER) and Golgi localization is altered in the absence of Alix and Syntenin-1. Cells expressing GFP-tagged APP $^{\text {swe }}$ were stained with an (a) Blue-white fluorescent ER marker, (b) RFP-tagged Golgi network marker, or (c) monodansylcadaverine (MDC) before live-cell imaging on a Zeiss LSM 880 confocal microscope. Images are representative across at least three independent experiments. Scale bar $=20 \mu \mathrm{m}$. Pearson's Correlation Coefficient (PCC) calculated from $\geq 10$ cells. One-way ANOVA: **, $p<0.01 ;{ }^{*}, p<0.05$

carrying vesicles may contribute to the propagation of amyloid secretion and plaque formation in the brain [27, 54], while apparently contradictory data suggest that neuronal EVs may aid in clearance of toxic proteins by microglia uptake [55] or by enzymatic degradation of amyloid beta [56]. Given the abundance of data describing the immunoregulatory effects of EVs, it is also possible that secreted vesicles contribute in part to the inflammatory response seen in AD. As others have alluded to [57], amyloid-containing vesicles may very well 


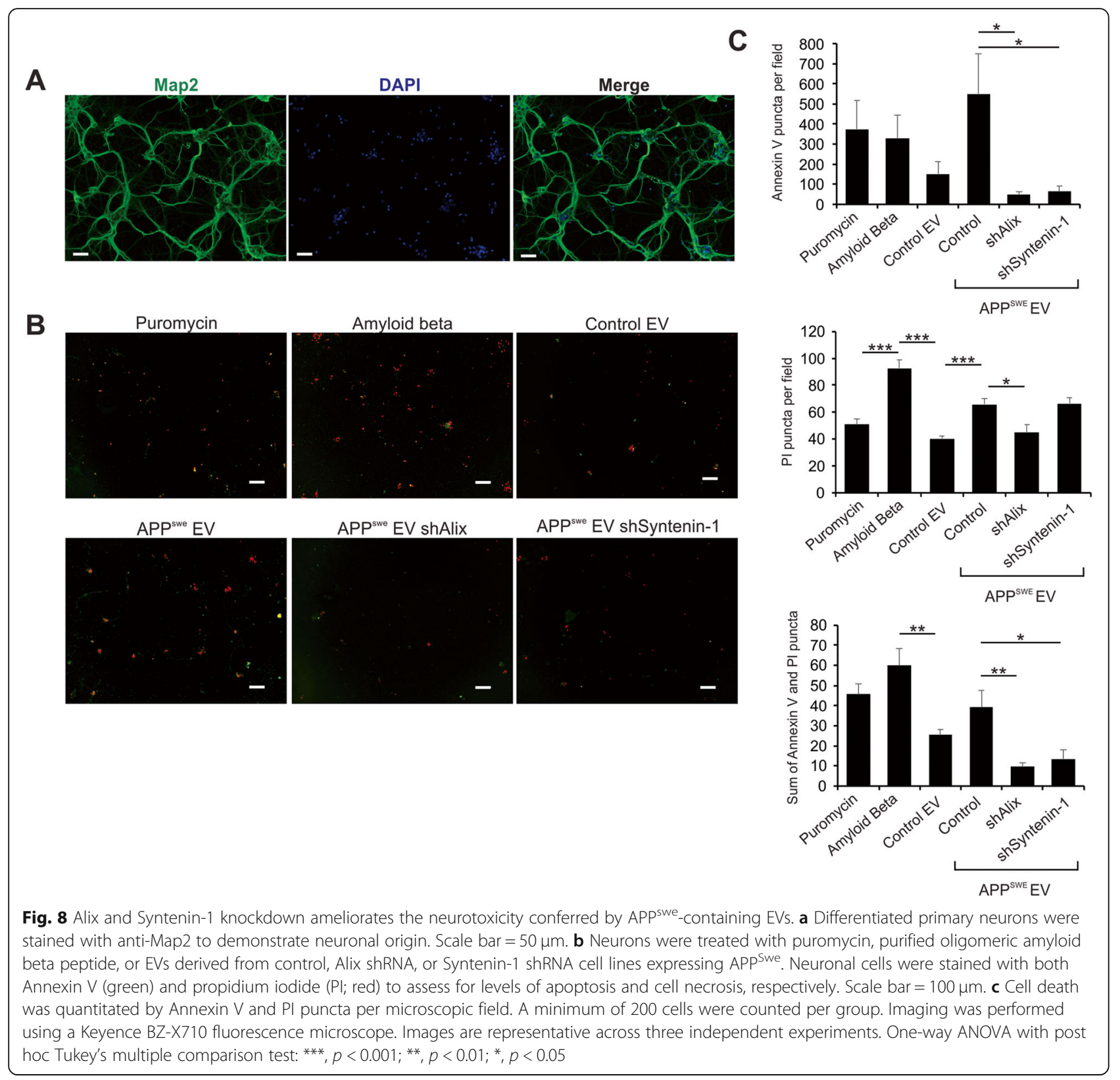

impart elements of both cellular protection and toxicity within the complexities of the neurodegenerative disease, aiding in clearance and sequestering (plaque development) of soluble amyloid from one neuron at the expense of uptake in another.

The C-terminal fragment of APP has been shown to be associated with amyloid plaques, and previous research has shown it accumulates in the brain of various mouse models [28]. Aggregation of CTF $\beta$ occurs mainly in endolysosomal compartments and may contribute to lysosomal dysfunction, one of the hallmarks of AD pathology $[24,25,58,59]$. Similar to $A \beta$, oligomerization of CTF $\beta$ can occur, and oligomers may be detected in secreted EVs [28]. The role of CTF $\beta$ in AD pathology could also explain some of the adverse side effects of $\gamma$ secretase inhibitor therapies for AD [24]. Of course, while we demonstrate that the predominant APP metabolite in EVs secreted from $\mathrm{APP}^{\text {swe }}$ transfected cells is likely CTF $\beta$, we do not rule out the possibility of a lower level of $A \beta$ or CTF $\alpha$ packaging.

In order to better understand the roles of pathogenic proteins in $\mathrm{AD}$ progression, an increased understanding of the mechanisms of intracellular and intercellular trafficking of these target proteins is necessary. Here, we demonstrate that a mutant amyloid precursor protein $\left(\mathrm{APP}^{\mathrm{swe}}\right)$ is trafficked into small EVs for secretion by an Alix-Syntenin-1 dependent mechanism, independent of early or late ESCRT machinery. It is likely that CD63 


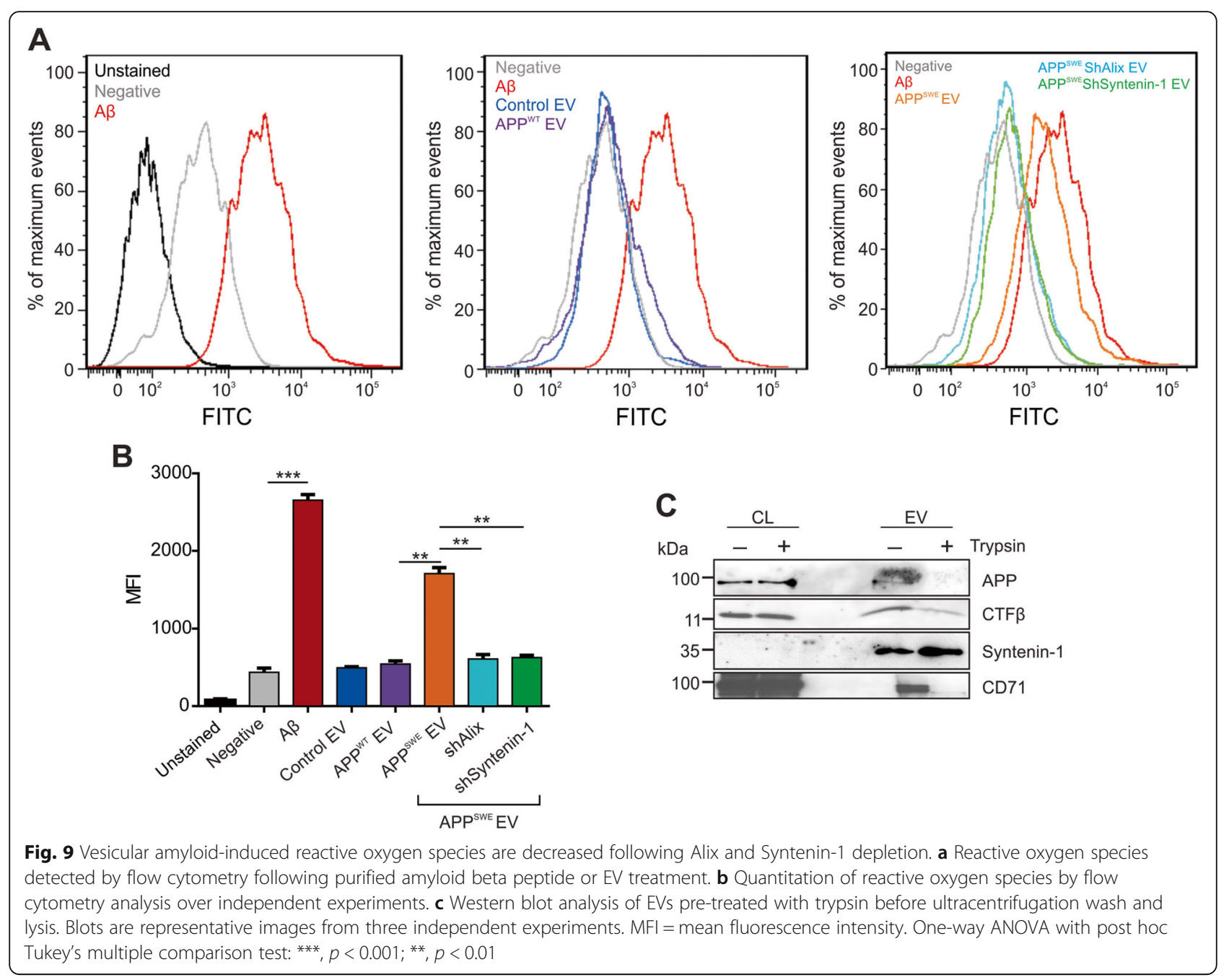

also engages in this ESCRT-independent pathway, as previously demonstrated [34]. This pathway appears to be conserved in terminally differentiated neuronal cells. Although this study is practically limited by the need to express high levels of exogenous APP, as vesicleassociated CTF $\beta$ is difficult to detect under endogenous conditions, a demonstrable decrease in endogenous SY5Y and HEK293 cell-derived EV APP was similarly observed. Of note, hampered EV production from cells lacking Alix and Syntenin-1 was not seen, and instead, detectable increases in vesicles were noted to be secreted from these cells. These findings suggest that the AlixSyntenin-1 pathway may be more involved in protein trafficking and cargo loading into vesicles, rather than EV budding or primary biogenesis, as has been previously suggested. It is possible that the augmented vesicle production may be compensatory in the setting of disrupted cargo packaging into individual EVs $[29,60]$.

Interestingly, while Alix and Syntenin-1 are necessary for proper intracellular trafficking of APP, early ESCRT proteins Hrs and Tsg101 appear to be additionally helpful in regulating the release of vesicular wild-type full length APP in this study. The discordance of early ESCR $\mathrm{T}$ involvement between wild-type and mutant APP trafficking is not completely clear. It is possible that APP ${ }^{\mathrm{WT}}$ requires ESCRT recognition of its ubiquitination status to guide sorting from the plasma membrane into endosomes, while $\mathrm{APP}^{\mathrm{swe}}$ bypasses this limitation. Future studies are required to elicit a better understanding of this mechanism [61-63].

Altered intracellular trafficking of APP clearly influences the secretion of the full-length and processed protein. It is possible that depletion of key proteins involved in vesicle biogenesis, including Alix and Syntenin-1, may lead to general disruption of endosomal compartment organization. However, decreased secretion of APP was seen following Alix and Syntenin-1 knockdowns in the absence of global vesicle reduction, suggesting a more specific interaction guiding intracellular APP trafficking. In the absence of Alix, APP was observed to be 
sequestered in perinuclear regions of the cell, primarily localized in the endoplasmic reticulum and Golgi. It has previously been proposed that Syntenin-1 binds syndecan assemblies on the surface of endosomal membranes; this complex subsequently recruits Alix to initiate processes involved in vesicle budding for ILV formation. The proposed order of these protein interactions is difficult to determine in the context of APP localization, as seen in this study. While the mechanism and significance of APP sequestration in the ER and Golgi is unclear, it is possible that this may in part reflect the importance of Alix-mediated formation of Golgi transport vesicles that bud from the endoplasmic reticulum, as previously described [64]. In this case, following Alix depletion, immature APP trafficking from the ER and Golgi may be limited. In contrast, APP localized predominately to lysosomes following Syntenin-1 knockdown. In the absence of the syndecan adaptor protein, Alix and Syntenin-1-mediated ILV formation is likely hampered, and APP is likely diverted to lysosomal compartments in lieu of incorporation into endosomal ILVs and extracellular vesicle secretion. Additional work will be necessary to unfold the protein-protein interactions driving these cellular pathways toward EV secretion.

Despite the unknown surrounding APP intracellular trafficking in the absence of Alix and Syntenin-1, the impact of these protein depletions on amyloid-mediated neurotoxicity was particularly pronounced in this study. Consistent with the reduction in APP and CTF $\beta$ secreted into EVs, the neurotoxic effect (by cell viability and ROS production assays) conferred by vesicles was similarly decreased following genetic knockdown of Alix and Syntenin-1. In comparison, transfer of $\mathrm{APP}^{\text {swe }}$-containing vesicles induced neuronal cell death comparable to amyloid beta peptide alone, suggesting that cleaved APP on the external surface of EVs is present in an active or accessible form, as has been suggested [53]. It is also possible that the neurotoxic effect may be in part mediated by non-APP related factors not examined in this study.

To our knowledge, ROS induction by CTF $\beta$ has not been well described. Here, we propose that EVassociated CTF $\beta$ causes a ROS response similarly to $A \beta$ peptide. Of note, we observed remnant detectable CTF $\beta$ following trypsin digestion of EV membrane proteins in comparison to near fully digested APP and CD71. It is plausible that this reflects relative protease resistance of CTF $\beta$, perhaps due to oligomerization on the surface of EVs, which is reduced on subsequent gel analysis. Previous evidence suggests that amyloid-induced neurotoxicity is mediated by binding of the peptide to amyloid receptors on the surface of recipient cells [46]. In this study, we demonstrate the predominant topographical distribution of both APP and CTF $\beta$ to the membrane or external surface of EVs, further supporting the ability of vesicles to carry these biologically active proteins. We also note that apoptotic cell death appears to dominate as the major mechanism of neuronal death conferred by amyloid carrying EVs while purified $A \beta$ peptide induces necrosis predominately. It is possible that $\mathrm{CTF} \beta$ mediates a separate neurotoxic effect compared to $A \beta$ that could be a result of its partial localization to mitochondria-associated ER membranes leading to mitochrondrial dysfunction [61]. Further work will be necessary to understand the mechanisms underlying these pathways.

\section{Conclusions}

A previous study by Dinkins et al. demonstrated that reduction of EVs in vivo by inhibition of ceramide production led to decreased amyloid plaque deposition and amelioration of the neurocognitive effects seen in an $\mathrm{AD}$ mouse model expressing a similar mutant APP [27]. Our present in vitro work supports the proposed neurotoxicity mediated by EVs containing mutant APP and CTF $\beta$, and further elucidates a mechanism of APP trafficking into small EVs for secretion. Here we use a targeted genetic approach to knockdown key proteins involved in APP intracellular trafficking, implicating the recently described Alix-Syntenin-1 pathway in APP sorting and vesicular secretion. Sphingomyelinase inhibitors have rather been shown to exhibit a global reduction of EV populations released from cells, the impact of which is unknown, particularly in the context of in vivo mouse studies. Our findings in this study provide tools to further, and more specifically, examine the impact of reducing APP vesicle secretion on the neurodegeneration and cognitive dysfunction seen in $\mathrm{AD}$ mouse models. These data may further shed light on the broader protein-protein interactions that guide vesicular protein trafficking and EV secretion, an area of interest that remains rather elusive across many fields of ongoing scientific research.

\section{Methods}

\section{Cell culture}

HEK293 (ATCC; CRL-1573) were cultured in Dulbecco modified Eagle medium (DMEM; Sigma; D5796). SHSY5Y cells (a gift from Cao Chuanhai, University of South Florida) were cultured in DMEM/ Ham's F-12 50/ 50 mix (DMEM/ F-12 50/50; Corning; 10-090-CV). Medium was supplemented with $10 \%$ fetal bovine serum (FBS; Seradigm; 1400-500), 2 mM L-glutamine (Corning; 25-005-CI), $100 \mathrm{IU}$ of penicillin-streptomycin (Corning; 30-002-CI), and $100 \mu \mathrm{g} / \mathrm{mL}: 0.25 \mu \mathrm{g} / \mathrm{mL}$ antibiotic/antimycotic (Corning; 30-004-CI). Serum used for experiments was depleted of extracellular vesicles by ultracentrifugation at $100,000 \times \mathrm{g}$ for $20 \mathrm{~h}$ and filtered 
through a $0.2-\mu \mathrm{m}$ filter prior to being added to culture medium. At the time of harvest, live cells were counted with an automated cell counter (Cellometer Vision, software version 2.1.4.2; Nexcelom Biosciences) by staining with $0.2 \%$ trypan blue (Sigma; T8154) in phosphatebuffered saline (PBS). Total live cell counts were used to derive particles per cell in subsequent nanoparticle tracking analyses.

\section{Primary cortical neuron preparation}

All animal procedures were carried out in accordance with the guidelines for the Florida State University Institutional Animal Care and Use Committee (ACUC) and all studies were performed in accordance with the recommendations in the National Institute of Health's Guide for the Care and Use of Laboratory Animals. Cultures of primary cortical neurons were prepared using a standard procedure. Briefly, cerebral cortices were dissected from postnatal day 0 mice with the aid of a stereo microscope. Isolated cerebral cortices were digested with papain (Worthington Biochemical Corporation; LS003119) for $5 \mathrm{~min}$ at $37^{\circ} \mathrm{C}$. Isolated neurons were seeded on poly-D-lysine-coated plates at a density of 470 cells $/ \mathrm{mm}^{2}$ and were cultured in neurobasal A medium (Gibco; A3653401) supplemented with B-27 (Gibco; A3582801), $0.5 \mathrm{mM} \mathrm{L-glutamine,} \mathrm{and} \mathrm{penicillin-}$ streptomycin at $37^{\circ} \mathrm{C}$ and $5 \% \mathrm{CO}_{2}$.

\section{Generation of shRNA constructs}

Oligonucleotides targeting ALIX, SDCBP (encoding Syntenin-1), TSG101, HRS, and CD63 genes were constructed using target sequences obtained from the Broad Institute GPP Web Portal. The control scramble sequence was generated using the Invivogen shRNA scramble tool as previously described [65]. The oligonucleotides listed below were annealed and ligated using T4 ligase between the Bgl II/Hind III sites of pENTR/ pTER+ (Addgene plasmid \# 17453, a gift from Eric Campeau \& Paul Kaufman). Entry clones were then sequenced for verification, and recombined with the destination vector pLenti X1 Zeo Dest (Addgene plasmid \# 17299, a gift from Eric Campeau \& Paul Kaufman) using LR recombination (Invitrogen \#11791-020) according to the manufacturer's instructions, and as previously described [65].

ALIX:

GATCCCGCATAATCAAGGCACTGTAAAGTGTGCT GTCCTTTACAGTGCCTTGATTATGCTTTTTGGAAA and AGCTTTTCCAAAAAGCATAATCAAGGCACTGTAAAG GACAGCACACTTTACAGTGCCTTGATTATGCGG.

SDCBP (Syntenin-1): GATCCCTATAGCATACTTGCAT CTTTAGTGTGCTGTCCTAAAGATGCAAGTATGCTAT ATTTTTGGAAA and AGCTTTTCCAAAAATATAGCAT

\section{ACTTGCATCTTTAGGACAGCACACAAAGATGCAAGT ATGCTATAGG. \\ TSG101: \\ GATCCCGTACGTCTTCTGTCCCGTAAAGTGTG \\ CTGTCCTTTACGGGACAGAAGACGTACTTT \\ TTGGAAA and. \\ AGCTTTTCCAAAAAGTACGTCTTCTGTCCCGTAAAG \\ GACAGCACACTTTACGGGACAGAAGACGTACGG. \\ CD63 (combined for lentivirus production): \\ GATCCCCAACGAGAAGGCGATCCATAAGTGTG CTGTCCTTATGGATCGCCTTCTCGTTGTCTTTTT \\ GGAAA and. \\ AGCTTTTCCAAAAACAACGAGAAGGCGATC CATAAGGACAGCACACTTATGGATCGCCTTCT \\ CGTTGGG.}

GATCCCTGGGATTAATTTCAACGAGAAGTGTG

CTGTCC TTCTCGTTGAAATTAATCCCATTTTT

GGAAA and.

AGCTTTTCCAAAAATGGGATTAATTTCAACGAGAAG

GACAGCACACTTCTCGTTGAAATTAATCCCAGG.

HRS:

GATCCCACGGTATCTCAACCGGAACTAGTGTG

CTGTCCTAGTTCCGGTTGAGATACCGTCTTTT

TGGAAA and.

AGCTTTTCCAAAAAACGGTATCTCAACCGG

AACTAGGACAGCACACTAGTTCCGGTTGAGAT

ACCGTGG.

Scramble:

GATCCCGAGCTTCGCGATCCAAGATAAGTGTG

CTGTCCTTATCTTGGATCGCGAAGCTCTTT

TTGGAAA and AGCTTTTCCAAAAAGAGCTTCG CGATCCAAGATAAGGACAGCACACTTATCTTG GATCGCGAAGCTCGG.

\section{Lentivirus production and stable cell generation}

Lentivirus particles for transduction and stable cell generation were produced in HEK293T cells following lipofectamine transfection of expression plasmids (pLenti CMV TetR BLAST and plenti X1 shRNA plasmids) and packaging plasmids pMD2.G (Addgene; number 12259; a gift from Didier Trono) and PSPAX2 (Addgene; number 12260; a gift from Didier Trono) according to the manufacturer's instructions (Invitrogen, L3000015). Of note, to increase efficiency of the knockdown, lentivirus particles for shRNA constructs targeting CD63 were generated by mixing two expression plasmids, generated by the dual oligonucleotides listed. Medium was collected at 48,72 , and $96 \mathrm{~h}$ post-transfection, centrifuged for $10 \mathrm{~min}$ at $1000 \times \mathrm{g}$, filtered through a $0.45-\mu \mathrm{m}$ filter, and frozen at $-80^{\circ} \mathrm{C}$ until use.

Cells stably expressing shRNA of different genes under the control of a tetracycline-inducible promoter were created by first transducing HEK293 or SH-SY5Y cells with lentivirus particles containing pLenti CMV TetR 
BLAST (Addgene; number 17492). Stable cells were selected with medium containing $10 \mu \mathrm{g} / \mathrm{mL}$ of blasticidin (Invivogen; ant-bl-1) and then transduced with plenti $\mathrm{X} 1 /$ zeo shHrs, plenti X1/zeo shAlix, plenti X1/zeo shSyntenin, plenti X1/zeo shTsg101, plenti X1/zeo shCD63, or plenti X1/zeo shScramble. Doubly stable cells were selected with medium supplemented with blasticidin $(10 \mu \mathrm{g} / \mathrm{mL})$ and zeocin $(200 \mu \mathrm{g} / \mathrm{mL})$ for 2 weeks.

\section{Transfection}

Plasmids containing wild-type human APP $\left(\mathrm{APP}^{\mathrm{WT}}\right)(\mathrm{p}-$ CAX APP 695) or human APP carrying the Swedish/Indiana mutations $\left(\mathrm{APP}^{\mathrm{swe}}\right)(\mathrm{pcAX}$ APP Swe/Ind) were purchased from Addgene (Addgene plasmid \#30137 and \#30145 respectively) [66]. The Vps4a and Vps4a E228Q constructs were generously gifted from the laboratory of Dr. Nicholas Buchkovich (Pennsylvania State University) [44]. GFP-tagged $\mathrm{APP}^{\text {swe }}$ was created by inserting the pCAX APP Swe/Ind plasmid into the pENTR1A-GFPN2 vector (Addgene \#19364) between the Hind III and Ncol restriction sites using T4 ligase. Entry clones were then sequenced for verification, and recombined with the destination vector $\mathrm{PQCXIZ} \mathrm{CMV/TO} \mathrm{DEST} \mathrm{destin-}$ ation vector (Addgene \#17401) using LR recombination (Invitrogen \#11791-020) according to the manufacturer's instructions, and as previously described [65].

HEK293 stably expressing silent shRNA constructs in $100 \mathrm{~mm}$ plates were induced by addition of $1 \mu \mathrm{g} / \mathrm{mL}$ of doxycycline $24 \mathrm{~h}$ before transfection with $5 \mu \mathrm{g}$ of APP 695 or APP Swe/Ind plasmid using Lipofectamine 3000 transfection kit (Invitrogen, L3000015). Lipofectamine reagents were diluted in Opti-MEM medium (Gibco, 31, 985-070) and added according to manufacturer's instructions. Twenty-four hours after transfection, cells were harvested and lysed in RIPA buffer, as previously described [40]. Cell-conditioned medium was harvested for extracellular vesicle enrichment.

For experiments involving SH-SY5Y cells, a similar protocol was followed. However, SH-SY5Y cells were seeded in plates coated with poly-lysine according to the Poly-L-Lysine Cell Attachment Protocol (Sigma) to ensure adequate adherence. Twenty-four hours later, cells were induced with $1 \mu \mathrm{g} / \mathrm{mL}$ of doxycycline to promote shRNA expression. To differentiate the neuroblast-like cells into a phenotype resembling mature neuronal cells, serum-containing medium was aspirated $24 \mathrm{~h}$ after induction and replaced with serum-free medium. In addition, $10 \mu \mathrm{M}$ of all-trans retinoic acid (Sigma, R2625) was added to cells, according to Shipley et al. [67] at the time of APP Swe/Ind plasmid transfection $24 \mathrm{~h}$ after induction. Forty-eight hours later, cell-conditioned medium was harvested for EV enrichment, and cell lysates were prepared as described below.

\section{Extracellular vesicle enrichment}

Extracellular vesicles were isolated from cell-conditioned medium by modified differential centrifugation involving a polyethylene glycol (PEG) precipitation/concentration step as previously described and extensively characterized [40, 68-70]. Briefly, medium was collected and centrifuged serially $(500 \mathrm{x}$ g for $5 \mathrm{~min}$; $2000 \mathrm{x} \mathrm{g}$ for $10 \mathrm{~min}$; $10,000 \mathrm{x} g$ for $30 \mathrm{~min}$ ) to remove larger contaminants, or collect pellets for Fig. 1a. To enhance the concentration and yield of small EVs, supernatants following the 10, $000 \mathrm{~g}$ spin were incubated with a 1:1 volume of $2 \times$ PEG solution $(16 \%$, wt/vol, polyethylene glycol, $1 \mathrm{M} \mathrm{NaCl})$ overnight. The next day, solutions were centrifuged at $3214 \mathrm{x} \mathrm{g}$ for $1 \mathrm{~h}$ to obtain crude EVs. Pellets were resuspended in phosphate-buffered saline (PBS) before an ultracentrifugation wash at $100,000 \times \mathrm{g}$ for $70 \mathrm{~min}$. EV pellets following ultracentrifugation were lysed in $2 \times$ nonreducing Laemmli sample buffer (4\% SDS, $100 \mathrm{mM}$ Tris- $\mathrm{HCl}$ [pH 6.8], $0.4 \mathrm{mg} / \mathrm{mL}$ bromophenol blue, $20 \%$ glycerol) for immunoblot analysis. Alternatively, pellets for nanoparticle tracking analysis were resuspended in particle-free PBS. For further purification and subpopulation separation by floatation density gradient, EV pellets following the $100,000 \mathrm{x}$ g spin were instead resuspended in $1.5 \mathrm{~mL}$ of $0.25 \mathrm{M}$ sucrose buffer $(10 \mathrm{mM}$ Tris [pH 7.4]). Gradients (10-30\%) were constructed as previously described in detail [40, 41, 43] using OptiPrep (Sigma, D1556). Following fractionation, densities of gradient separated fractions were estimated by measuring refractive indices of fractions with a refractometer (Refracto 30PX). Samples were then washed in PBS and pelleted again by ultracentrifugation at 100,000 $\mathrm{xg}$ for 2 h. Final pellets were resuspended in particle-free PBS for electron microscopy. A 1:1 solution of strong ureacontaining lysis buffer (5\% SDS, $10 \mathrm{mM}$ EDTA, $120 \mathrm{mM}$ Tris- $\mathrm{HCl}$ [pH 6.8], $8 \mathrm{M}$ urea) with the addition of a protease inhibitor cocktail (Thermo, 78,438) was added to samples in fractions 2-6 for immunoblot analysis. EV characterization was performed in accordance with the minimal information for studies of EVs (MISEV) 2018 guidelines issued by the International Society for Extracellular Vesicles [71, 72].

\section{Electron microscopy}

Electron microscopy was conducted on EV samples isolated in vesicle-rich fractions of the iodixanol density gradient purification step. Grids were prepared and images were obtained as previously described [73].

\section{Nanoparticle tracking analysis}

Nanoparticle tracking was performed using a Malvern NanoSight LM10 instrument, and videos were processed using NTA 3.1 software as previously described [74]. 


\section{Immunoblot analysis}

Whole-cell lysates were prepared by washing cells and then scraping them into cold PBS before pelleting at $1000 \times \mathrm{g}$ for $10 \mathrm{~min}$ and lysis by radioimmunoprecipitation assay (RIPA) buffer as described previously [40]. To prepare all cell and EV lysates run under reducing conditions for SDS-PAGE, additional sample buffer $(5 \times)$ also containing 0.2 M dithiothreitol (DTT) and 2\% BME was added to samples. Equal protein of cell lysates measured by Pierce $660 \mathrm{~nm}$ Protein Assay (Invitrogen, 22,662) or equal volume of EV samples was loaded into an SDS 10, $12 \%$, or $15 \%$ polyacrylamide gel. Western blot analysis was performed as described [40]. Ponceau S stain was used to visualize total protein. Blots were probed using the following antibodies: Alix (Q-19; Santa Cruz Biotechnology, 2171; Cell Signaling), Hsc70 (B-6; Santa Cruz), Tsg101 (C-2; Santa Cruz, 4A10; Genetex), CD63 (TS63; Abcam), Hrs (M-79; Santa Cruz), APP (LN27; Biolegend, 2452; Cell Signaling), Amyloid beta (8243, 2454; Cell Signaling), CD9 (P1/33/2; Santa Cruz, MM2/ 57; Millipore), Syntenin-1 (S-31; Santa Cruz), Flotillin-2 (H-90; Santa Cruz), GFP (600-101-215; Rockland), CD81 (H-121; Santa Cruz), Calnexin (H-70; Santa Cruz), CD71 (13,113; Cell Signaling), APP-CTF (A8717; Millipore), $\beta$-Amyloid 1-16 (6E10; Biolegend), rabbit antimouse IgG (Genetex, 26,728), rabbit anti-goat IgG (Genetex, 26,741), or goat anti-rabbit IgG (Fab fragment) (Genetex, 27,171). Blots were imaged using an Image Quant LAS4000 (General Electric) and processed with ImageQuant TL v8.1.0.0 software, Adobe Photoshop CS6, and CorelDraw Graphic Suite X5.

\section{Trypsin treatment of EVs}

For experiments in Fig. 9c-d, PBS-suspended EVs from cells transfected with $\mathrm{APP}^{\text {swe }}$ were subjected to trypsin pre-treatment. Vesicles were incubated with $0.25 \%$ trypsin EDTA (Corning, 25-053-CI) or PBS with $2.21 \mathrm{mM}$ EDTA (Thermo $1,861,275$ ) for $20 \mathrm{~min}$ at $37^{\circ} \mathrm{C}$. EVs were then washed with PBS and ultracentrifuged at 100,000 $\mathrm{x}$ $\mathrm{g}$ to re-pellet the vesicles before lysis with $0.1 \%$ SDS and protein quantification. Treated vesicle proteins were analyzed by conventional western blot analysis or dot blot analysis.

\section{Immunofluorescence assay}

HEK293 cells stably expressing shRNA plasmids targeting a scrambled control, Alix, or Syntenin-1 were seeded in 6-well plates on square glass coverslips. Cells were induced with $1 \mu \mathrm{g} / \mathrm{mL}$ doxycycline five hours later. Cells were transfected with $2 \mu \mathrm{g}$ of APP Swe/Ind plasmid DNA using Lipofectamine 3000 (Invitrogen) 20-24h after initial seeding. Twenty-four hours after transfection, medium was aspirated, and cells were gently washed with PBS. Cells were fixed on the coverslip in
4\% paraformaldehyde for $10 \mathrm{~min}$, then washed again in PBS before permeabilization in $0.2 \%$ Triton X-100 (in PBS) for $30 \mathrm{~min}$ at room temperature. A $0.2 \%$ Tween solution in PBS was prepared to make PBS-T. Cells were blocked in 5\% goat serum/PBS-T for another $30 \mathrm{~min}$ at room temperature, then primary anti-APP antibody (2452; Cell Signaling) was added for a 3-h room temperature incubation. Cells were washed with PBS before secondary anti-rabbit antibody in 5\% goat serum/ PBS- $T$ was added for $1 \mathrm{~h}$ at room temperature, then subsequently washed with PBS, and incubated with DAPI stain (Thermo, 62,248) in PBS for 10 min. Finally, cells were washed once more with PBS and mounted on a glass slide with mounting medium (4\% propyl gallate, 90\% glycerol in PBS) for confocal microscopy imaging. Confocal images were taken using a Zeiss LSM 880 microscope with 488-nm and 405-nm lasers and processed using Zen 2.1 Black software.

Primary neuronal cultures at days in vitro (DIV) 9 were stained with an antibody against microtubule associated protein 2 (Map2), a neuronal cell body and neurite marker. Cells were grown on poly-D-lysine-coated glass coverslips and were fixed in $4 \%$ paraformaldehyde and $4 \%$ sucrose in PBS for $20 \mathrm{~min}$ at room temperature, then blocked in a PBS solution containing 0.3\% Triton $\mathrm{X}-100$ (PBST) and 5\% normal goat serum for $30 \mathrm{~min}$ at room temperature. Cells were then incubated in $1 \%$ normal goat serum in PBS containing anti-MAP2 antibody (17490-1-AP, ProteinTech, 1:250 dilution) at $4{ }^{\circ} \mathrm{C}$ overnight. The next day, sections were washed with PBS $3 \times$ 5 min, followed by incubation with FITC-conjugated secondary antibody (4050-02, Southern Biotech, 1:1000 dilution) for $2 \mathrm{~h}$ at room temperature. After several washes with PBS, the coverslips were counterstained with $5 \mu \mathrm{g} /$ $\mathrm{mL}$ DAPI (Sigma-Aldrich) in PBS for $5 \mathrm{~min}$, washed in PBS for 5 min and mounted with Vectashield (Vector laboratories) to retard fluorescence fading. Images of cells were obtained using a Keyence BZ-X710 fluorescent microscope.

\section{Live cell imaging}

For subcompartmental localization analysis of APP ${ }^{\text {swe }}$, shRNA-containing HEK293 cells were seeded into 35 $\mathrm{mm}$ glass bottom dishes (Greiner, 627,860) and induced with doxycycline the next day, as described above. Approximately $4 \mathrm{~h}$ later, cells were transfected with $2 \mu \mathrm{g}$ of GFP-tagged APP using lipofectamine transfection reagent, and incubated for another $24 \mathrm{~h}$. Staining with cellular compartment specific stains was performed as follows. Lissamine ${ }^{\mathrm{T} x}$ Rhodamine B 1,2-Dihexadecanoylsn-Glycero-3-Phosphoethanolamine, Triethylammonium Salt (rhodamine DHPE; Thermo Fisher; L1392) was used to stain endosomal compartments as previously described [75]. Briefly, stock DHPE was dissolved in 
chloroform for a concentration of $5 \mathrm{mg} / \mathrm{mL}$. One $\mu \mathrm{L}$ of stock was resuspended in $50 \mu \mathrm{L}$ of cold $95 \%$ ethanol, then further diluted 1:600 in cold PBS, before adding $200 \mu \mathrm{L}$ to cells in a dropwise manner and incubating for $30 \mathrm{~min}$. Cells were washed and double stained with Hoechst nuclear stain $(5 \mu \mathrm{g} / \mathrm{mL} ; 62,249 ;$ Thermo Scientific) before live-cell imaging. LysoTracker ${ }^{\mathrm{Tm}}$ Red DND-99 (Thermo Fisher; L7528) was used to highlight lysosomal compartments by incubated cells with $75 \mathrm{nM}$ of the LysoTracker stain before washing and adding Hoechst nuclear stain for $30 \mathrm{~min}$. For Golgi staining, cells were incubated with CellLight ${ }^{\circ}$ Golgi-RFP, BacMam 2.0 (15 particles per cell; Thermo Fisher; C10593) $16 \mathrm{~h}$ before washing, staining with Hoechst nuclear stain, and imaging. For ER staining, $1 \mu \mathrm{M}$ final concentration of ER-Tracker $^{\text {Tu }}$ Blue-White DPX (Thermo Fisher; E12353) was added to cells for $30 \mathrm{~min}$ before cells were washed and imaged. To visualize autophagic vacuoles within cells, Mono-dansylcadaverine (MDC; 30,432; Sigma) was added to cells at a final concentration of $50 \mathrm{nM}$ for 30 min before washing cells and imaging as previously described [69]. All images were taken using a Zeiss LSM 880 microscope with 543-nm, 488-nm, and 405-nm lasers and processed using Zen 2.1 Black software.

\section{Neurotoxicity assay}

Following growth in neurobasal A medium, primary cortical neurons were treated with $2 \mu \mathrm{g} / \mathrm{mL}$ puromycin, $0.25 \mu \mathrm{M}$ purified oligomerized amyloid beta (see reactive oxygen species detection methods below), or $20 \mu \mathrm{g}$ of EVs isolated from respective cell lines by PEG precipitation and subsequent ultracentrifugation wash. Fortyeight hours after treatment, medium was aspirated and cells were scraped into cold PBS and centrifuged at 300 $\mathrm{xg}$ for $2 \mathrm{~min}$. Cells were resuspended in $100 \mu \mathrm{L}$ of Annexin $\mathrm{V}$ binding buffer containing $0.25 \mu \mathrm{L}$ Annexin V-FITC and $0.25 \mu \mathrm{L}$ ViaStain $^{\text {ma }}$ propidium iodide (PI) stain (Biolegend, 422,201; Biolegend, 640,905; and Nexcelom, CSK-0112) then incubated in the dark for 10 min. Cells were centrifuged again at $300 \mathrm{~g}$ for $2 \mathrm{~min}$, and resuspended in $500 \mu \mathrm{L}$ of Annexin $\mathrm{V}$ binding buffer (without stains). Images were taken on a Keyence BZX710 fluorescent microscope.

Puncta Analyzer plugin was used as described previously [76] to quantify individual puncta for Annexin V and PI stained cells. Five 10x images per condition were analyzed with a minimum of 200 cells counted per group. With region of interest (ROI) selected as the full 10x image, the Puncta Analyzer plugin was used to analyze Red channel (PI) and Green Channel (Annexin V) following background subtraction (rolling ball radius of 50, without white background). Threshold remained consistent across groups, and the minimum puncta size was set to 4 pixels. Data are displayed as puncta per microscopic field normalized to the total number of neuronal cells per field.

\section{Undifferentiated hiPSC culture and cortical spheroid differentiation}

Human iPSK3 cells were maintained in mTeSR serumfree medium (StemCell Technologies, Inc., Vancouver, Canada) on 6-well plates coated with growth factor reduced Geltrex (Life Technologies, Carlsbad, CA) as previously reported [77]. The cells were passaged by Accutase dissociation every seven days and seeded at $1 \times 10^{6}$ cells per well of tissue culture treated 6-well plate (in $3 \mathrm{~mL}$ medium) in the presence of $10 \mu \mathrm{M}$ Y27632 (Sigma) for the first 24h [77-79]. For neural differentiation, human iPSK3 cells were seeded into Ultra-Low Attachment 24-well plates (Corning Incorporated, Corning, NY) at $3 \times 10^{5}$ cells/well in $1 \mathrm{~mL}$ of differentiation medium composed of Dulbecco's Modified Eagle Medium/Nutrient Mixture F-12 (DMEM/F-12) plus 2\% B-27 serum-free supplement (Life Technologies). Y27632 $(10 \mu \mathrm{M})$ was added during the seeding and removed after $24 \mathrm{~h}$. On day 1 , the cells formed spheroids and were treated with dual SMAD signaling inhibitors $10 \mu \mathrm{M}$ SB431542 (Sigma) and $100 \mathrm{nM}$ LDN193189 (Sigma) $[77,79,80]$. After 8 days, the cells were treated with fibroblast growth factor (FGF)-2 $(10 \mathrm{ng} / \mathrm{mL}$, Life Technologies) and cyclopamine $(1 \mu \mathrm{M}$, Sigma) until day 24-30. Next day, the spheroids were re-plated to Geltrex-coated plates and grown for 3-5 days before the treatments.

\section{Reactive oxygen species detection}

To prepare oligomers of the $A \beta 42$ peptide, biotinylated A 342 (Bachem) was fully dissolved at $0.5 \mathrm{mg} / \mathrm{mL}$ in hexafluor-2-propanole (HFIP, Sigma) [77, 81]. HFIP A $\beta$ (1-42) solution was dispensed at $10 \mu \mathrm{L}$ into each siliconized Snap-Cap microtube. The microtubes were put in a desiccator to completely evaporate HFIP and thereafter stored at $-80^{\circ} \mathrm{C}$. Oligomer solutions were prepared freshly for each experiment. The stock was dissolved in $10 \mu \mathrm{L}$ of DMSO (to $105 \mu \mathrm{M}$ ) and incubated for $3 \mathrm{~h}$ at room temperature. Oligomers of $A \beta 42$ were added to the day 27-35 cortical spheroid cultures at $1 \mu \mathrm{M}$, as previous published [77, 81], and incubated for $48 \mathrm{~h}$ before analyzing. EVs $(20 \mu \mathrm{g})$ harvested from control HEK293 cells, and cell lines expressing $\mathrm{APP}^{\mathrm{WT}}$ or $\mathrm{APP}^{\text {swe }}$ in the absence or presence of concomitant shRNA targeting Alix or Syntenin-1 were added to spheroid cultures of the same maturity, then incubated for $48 \mathrm{~h}$. Reactive oxygen species (ROS) detection was performed using Image-iT ${ }^{\mathrm{TM}}$ Live Green Reactive Oxygen Species Detection kit (Molecular probes), as previously described [82]. Briefly, the cells were harvested and washed in Hank's Balanced Salt Solution, and incubated in a solution of 
$25 \mu \mathrm{M}$ carboxy- $\mathrm{H}_{2} \mathrm{DCFDA}$ for $30 \mathrm{~min}$ at $37^{\circ} \mathrm{C}$. The samples were then washed and analyzed by flow cytometry. The cultures treated with $A \beta 42$ oligomers were used as the positive control. The cultures that did not receive any treatment were used as the negative control.

\section{Data analysis and statistics}

Statistical analysis was performed using the GraphPad Prism 8 (GraphPad Software, San Diego, CA) or Microsoft Excel with a significant threshold of $p \leq 0.05$. All results are presented as means \pm standard error of means (SEMs). The statistical significance of differences between means was assessed using the one-way ANOVA with a post hoc Tukey's multiple comparison test or student's t-test. Pearson correlation coefficient (PCC) was determined using an ImageJ colocalization plugin, with a minimum of 10 cells analyzed. Analysis was performed using GraphPad Prism 8 was used to determine significance using a one-way ANOVA with a post hoc Tukey's multiple comparison test.

\section{Supplementary information}

Supplementary information accompanies this paper at https://doi.org/10. 1186/s12860-020-00302-0.

Additional file 1. Uncropped images of immunblots used in figures.

\begin{abstract}
Abbreviations
APP: Amyloid Precursor Protein; CTFa: Alpha C-terminal fragment; CTF $\beta$ : Beta C-terminal fragment; $A \beta$ : Amyloid beta; EVs: Extracellular vesicles;

AD: Alzheimer's disease; ESCRT: Endosomal sorting complexes required for transport; BACE: Beta-secretase; Hrs: Hepatocyte growth factor-regulated tyrosine kinase substrate; Stam: Signal transducing adaptor molecule; Tsg101: Tumor susceptibility gene 101; MVB: Multivesicular body; ILV: Intraluminal vesicles; WT: Wild-type; Swe: Swedish/Indiana mutation; KD: Knockdown; DMEM: Dulbecco modified Eagle medium; ACUC: Animal Care and Use Committee; RIPA: Radioimmunoprecipitation assay; PEG: Polyethylene glycol; PBS: Phosphate-buffered saline; MISEV: Minimal information for studies of EVs; TBS-T: Tris-buffered saline with Tween 20; DHPE: 1,2-Dihexadecanoyl-sn-Glycero-3-Phosphoethanolamine, Triethylammonium Salt; PI: Propidium iodide; MDC: Monodansylcadaverine; ROS: Reactive oxygen species; iPSK3: Human induced pluripotent stem cells
\end{abstract}

\section{Acknowledgements}

We thank Dingani Nkosi for his assistance in preparation of the shRNAexpressing cell lines used throughout this study, Michael Vreones for help in immunoblot analyses, Sara York and the Biological Science Imaging Resource at Florida State University for technical assistance with electron microscopy, and Mark Marzano for aid in the reactive oxygen species assays. Confocal imaging was performed with the support of the Florida State University College of Medicine Confocal Microscopy Laboratory.

\section{Authors' contributions}

ASC, SNH, and DGM conceived of and designed the experiments detailed in this study. YZ and YL aided with experimental design. ASC performed the majority of the experiments, with assistance from SNH. GSL performed and analyzed the primary cortical neuron toxicity experimental data, while XY performed the neuronal spheroid ROS studies. The manuscript was written by SNH, with editing contributions from ASC, YZ, YL, GSL, and DGM. DGM supervised the project and obtained the funding support. All authors read and approved the final manuscript.

\section{Funding}

This study was supported by a grant from the Florida Department of Health Ed and Ethel Moore Alzheimer's Disease Research Program awarded (6AZ11) and supplemental funds provided through the Alzheimer's Disease Initiative by the National Cancer Institute of the National Institutes of Health under Award Number R01CA204621 awarded to DGM. The content is solely the responsibility of the authors and does not necessarily represent the official views of the National Institutes of Health.

\section{Availability of data and materials}

The datasets used and/or analyzed during the current study are available from the corresponding author upon reasonable request.

\section{Ethics approval and consent to participate}

All animal procedures were carried out in accordance with the guidelines for the Florida State University Institutional Animal Care and Use Committee (ACUC) and all studies were performed in accordance with the recommendations in the National Institute of Health's Guide for the Care and Use of Laboratory Animals.

\section{Consent for publication}

Not applicable.

\section{Competing interests}

The authors declare that they have no competing interests.

\section{Author details}

${ }^{1}$ Department of Biomedical Sciences, Florida State University College of Medicine, 1115 West Call Street, Tallahassee, FL 32306-4300, USA.

${ }^{2}$ Department of Chemical and Biomedical Engineering, Florida State University, Tallahassee, FL, USA.

Received: 22 January 2020 Accepted: 16 July 2020 Published online: 30 July 2020

\section{References}

1. Busciglio J, Lorenzo A, Yankner BA. Methodological variables in the assessment of beta amyloid neurotoxicity. Neurobiol Aging. 1992;13(5):60912.

2. Pike CJ, Burdick D, Walencewicz AJ, Glabe CG, Cotman CW. Neurodegeneration induced by beta-amyloid peptides in vitro: the role of peptide assembly state. J Neurosci. 1993;13(4):1676-87.

3. Mullan M, Crawford F, Axelman K, Houlden H, Lilius L, Winblad B, et al. A pathogenic mutation for probable Alzheimer's disease in the APP gene at the N-terminus of beta-amyloid. Nat Genet. 1992;1(5):345-7.

4. Schaeffer EL, Figueiro M, Gattaz WF. Insights into Alzheimer disease pathogenesis from studies in transgenic animal models. Clinics (Sao Paulo). 2011;66(Suppl 1):45-54.

5. Murrell JR, Hake AM, Quaid KA, Farlow MR, Ghetti B. Early-onset Alzheimer disease caused by a new mutation (V717L) in the amyloid precursor protein gene. Arch Neurol. 2000;57(6):885-7.

6. Hall AM, Roberson ED. Mouse models of Alzheimer's disease. Brain Res Bull. 2012;88(1):3-12.

7. Rohan de Silva HA, Jen A, Wickenden C, Jen LS, Wilkinson SL, Patel AJ. Cellspecific expression of beta-amyloid precursor protein isoform mRNAs and proteins in neurons and astrocytes. Brain Res Mol Brain Res. 1997;47(1-2): 147-56.

8. Kang J, Müller-Hill B. Differential splicing of Alzheimer's disease amyloid A4 precursor RNA in rat tissues: PreA4(695) mRNA is predominantly produced in rat and human brain. Biochem Biophys Res Commun. 1990;166(3):1192200.

9. Furukawa K, Sopher BL, Rydel RE, Begley JG, Pham DG, Martin GM, et al. Increased activity-regulating and neuroprotective efficacy of alphasecretase-derived secreted amyloid precursor protein conferred by a Cterminal heparin-binding domain. J Neurochem. 1996;67(5):1882-96.

10. Mattson MP. Cellular actions of beta-amyloid precursor protein and its soluble and fibrillogenic derivatives. Physiol Rev. 1997;77(4):1081-132.

11. Parvathy S, Hussain I, Karran EH, Turner AJ, Hooper NM. Cleavage of Alzheimer's amyloid precursor protein by alpha-secretase occurs at the surface of neuronal cells. Biochemistry. 1999;38(30):9728-34. 
12. Haass C, Hung AY, Schlossmacher MG, Oltersdorf T, Teplow DB, Selkoe DJ. Normal cellular processing of the beta-amyloid precursor protein results in the secretion of the amyloid beta peptide and related molecules. Ann N Y Acad Sci. 1993:695:109-16.

13. Evrard C, Kienlen-Campard P, Coevoet M, Opsomer R, Tasiaux B, Melnyk P, et al. Contribution of the Endosomal-Lysosomal and proteasomal Systems in Amyloid- $\beta$ Precursor Protein Derived Fragments Processing. Front Cell Neurosci. 2018;12:435.

14. Andrew RJ, Kellett KA, Thinakaran G, Hooper NM. A Greek tragedy: the growing complexity of Alzheimer amyloid precursor protein proteolysis. J Biol Chem. 2016:291(37):19235-44.

15. Ehehalt R, Keller P, Haass C, Thiele C, Simons K. Amyloidogenic processing of the Alzheimer beta-amyloid precursor protein depends on lipid rafts. J Cell Biol. 2003;160(1):113-23.

16. Koo EH, Squazzo SL. Evidence that production and release of amyloid betaprotein involves the endocytic pathway. J Biol Chem. 1994;269(26):17386-9.

17. Kinoshita A, Fukumoto $H$, Shah $T$, Whelan CM, Irizarry MC, Hyman BT. Demonstration by FRET of BACE interaction with the amyloid precursor protein at the cell surface and in early endosomes. J Cell Sci. 2003;116(Pt 16):3339-46.

18. Fukumori A, Okochi M, Tagami S, Jiang J, Itoh N, Nakayama T, et al. Presenilin-dependent gamma-secretase on plasma membrane and endosomes is functionally distinct. Biochemistry. 2006;45(15):4907-14.

19. Harris B, Pereira I, Parkin E. Targeting ADAM10 to lipid rafts in neuroblastoma SH-SY5Y cells impairs amyloidogenic processing of the amyloid precursor protein. Brain Res. 2009;1296:203-15.

20. Laulagnier K, Javalet C, Hemming FJ, Chivet M, Lachenal G, Blot B, et al. Amyloid precursor protein products concentrate in a subset of exosomes specifically endocytosed by neurons. Cell Mol Life Sci. 2018;75(4):757-73.

21. Zheng $T$, Wu $X$, Wei $X$, Wang $M$, Zhang $B$. The release and transmission of amyloid precursor protein via exosomes. Neurochem Int. 2018;114: $18-25$.

22. Vingtdeux V, Hamdane $M$, Loyens A, Gelé P, Drobeck H, Bégard S, et al. Alkalizing drugs induce accumulation of amyloid precursor protein byproducts in luminal vesicles of multivesicular bodies. J Biol Chem. 2007; 282(25):18197-205

23. Rajendran L, Honsho M, Zahn TR, Keller P, Geiger KD, Verkade P, et al. Alzheimer's disease beta-amyloid peptides are released in association with exosomes. Proc Natl Acad Sci U S A. 2006;103(30):11172-7.

24. Lauritzen I, Pardossi-Piquard R, Bourgeois A, Pagnotta S, Biferi MG, Barkats M, et al. Intraneuronal aggregation of the $\beta$-CTF fragment of APP (C99) induces A $\beta$-independent lysosomal-autophagic pathology. Acta Neuropathol. 2016; 132(2):257-76.

25. Lauritzen I, Pardossi-Piquard R, Bauer C, Brigham E, Abraham JD, Ranaldi S, et al. The $\beta$-secretase-derived $C$-terminal fragment of $\beta A P P, C 99$, but not $A \beta$, is a key contributor to early intraneuronal lesions in triple-transgenic mouse hippocampus. J Neurosci. 2012;32(46):16243-55a

26. Pera M, Alcolea D, Sánchez-Valle R, Guardia-Laguarta C, Colom-Cadena M, Badiola N, et al. Distinct patterns of APP processing in the CNS in autosomal-dominant and sporadic Alzheimer disease. Acta Neuropathol. 2013;125(2):201-13.

27. Dinkins MB, Dasgupta S, Wang G, Zhu G, Bieberich E. Exosome reduction in vivo is associated with lower amyloid plaque load in the 5XFAD mouse model of Alzheimer's disease. Neurobiol Aging. 2014; 35(8):1792-800

28. Lauritzen I, Bécot A, Bourgeois A, Pardossi-Piquard R, Biferi MG, Barkats M, et al. Targeting $\gamma$-secretase triggers the selective enrichment of oligomeric APP-CTFs in brain extracellular vesicles from Alzheimer cell and mouse models. Transl Neurodegener. 2019;8:35

29. Colombo M, Moita C, van Niel G, Kowal J, Vigneron J, Benaroch P, et al. Analysis of ESCRT functions in exosome biogenesis, composition and secretion highlights the heterogeneity of extracellular vesicles. J Cell Sci. 2013;126(Pt 24):5553-65.

30. Babst M, Katzmann DJ, Snyder WB, Wendland B, Emr SD. Endosomeassociated complex, ESCRT-II, recruits transport machinery for protein sorting at the multivesicular body. Dev Cell. 2002;3(2):283-9.

31. Hurley JH. ESCRT complexes and the biogenesis of multivesicular bodies Curr Opin Cell Biol. 2008:20(1):4-11.

32. Roxrud I, Stenmark H, Malerød L. ESCRT \& co. Biol Cell. 2010;102(5):293-318.

33. Henne WM, Buchkovich NJ, Emr SD. The ESCRT pathway. Dev Cell. 2011; 21(1):77-91
34. Baietti MF, Zhang Z, Mortier E, Melchior A, Degeest G, Geeraerts A, et al. Syndecan-syntenin-ALIX regulates the biogenesis of exosomes. Nat Cell Biol. 2012;14(7):677-85

35. Roucourt B, Meeussen S, Bao J, Zimmermann P, David G. Heparanase activates the syndecan-syntenin-ALIX exosome pathway. Cell Res. 2015; 25(4):412-28.

36. Trajkovic K, Hsu C, Chiantia S, Rajendran L, Wenzel D, Wieland F, et al. Ceramide triggers budding of exosome vesicles into multivesicular endosomes. Science. 2008;319(5867):1244-7.

37. Escola JM, Kleijmeer MJ, Stoorvogel W, Griffith JM, Yoshie O, Geuze HJ. Selective enrichment of tetraspan proteins on the internal vesicles of multivesicular endosomes and on exosomes secreted by human Blymphocytes. J Biol Chem. 1998;273(32):20121-7.

38. Andreu Z, Yáñez-Mó M. Tetraspanins in extracellular vesicle formation and function. Front Immunol. 2014:5:442.

39. van Niel G, Charrin S, Simoes S, Romao M, Rochin L, Saftig P, et al. The tetraspanin CD63 regulates ESCRT-independent and -dependent endosomal sorting during melanogenesis. Dev Cell. 2011;21(4):708-21.

40. Hurwitz SN, Nkosi D, Conlon MM, York SB, Liu X, Tremblay DC, et al. CD63 Regulates Epstein-Barr Virus LMP1 Exosomal Packaging, Enhancement of Vesicle Production, and Noncanonical NF-KB Signaling. J Virol. 2017;91(5).

41. Kowal J, Arras G, Colombo M, Jouve M, Morath JP, Primdal-Bengtson B, et al. Proteomic comparison defines novel markers to characterize heterogeneous populations of extracellular vesicle subtypes. Proc Natl Acad Sci U S A. 2016; 113(8):E968-77.

42. Hurwitz SN, Sun L, Cole KY, Ford CR, Olcese JM, Meckes DG. An optimized method for enrichment of whole brain-derived extracellular vesicles reveals insight into neurodegenerative processes in a mouse model of Alzheimer's disease. J Neurosci Methods. 2018;307:210-20.

43. Hurwitz SN, Olcese JM, Meckes DG. Extraction of extracellular vesicles from whole tissue. J Vis Exp. 2019;144.

44. Streck NT, Carmichael J, Buchkovich NJ. Nonenvelopment Role for the ESCRT-III Complex during Human Cytomegalovirus Infection. J Virol. 2018; 92(12).

45. Manoharan S, Guillemin GJ, Abiramasundari RS, Essa MM, Akbar M, Akbar MD. The role of reactive oxygen species in the pathogenesis of Alzheimer's disease, Parkinson's disease, and Huntington's disease: a mini review. Oxidative Med Cell Longev. 2016;2016:8590578.

46. Kam TI, Gwon Y, Jung YK. Amyloid beta receptors responsible for neurotoxicity and cellular defects in Alzheimer's disease. Cell Mol Life Sci. 2014;71(24):4803-13.

47. Bellingham SA, Guo BB, Coleman BM, Hill AF. Exosomes: vehicles for the transfer of toxic proteins associated with neurodegenerative diseases? Front Physiol. 2012;3:124.

48. Cheng $L$, Zhao W, Hill AF. Exosomes and their role in the intercellular trafficking of normal and disease associated prion proteins. Mol Asp Med. 2017.

49. Guo BB, Bellingham SA, Hill AF. Stimulating the release of Exosomes increases the intercellular transfer of prions. J Biol Chem. 2016;291(10):512837.

50. Howitt J, Hill AF. Exosomes in the pathology of neurodegenerative diseases. J Biol Chem. 2016;291(52):26589-97.

51. Saman S, Kim W, Raya M, Visnick Y, Miro S, Jackson B, et al. Exosomeassociated tau is secreted in tauopathy models and is selectively phosphorylated in cerebrospinal fluid in early Alzheimer disease. J Biol Chem. 2012;287(6):3842-9.

52. Sardar Sinha M, Ansell-Schultz A, Civitelli L, Hildesjö C, Larsson M, Lannfelt L, et al. Alzheimer's disease pathology propagation by exosomes containing toxic amyloid-beta oligomers. Acta Neuropathol. 2018;136(1):41-56.

53. Eitan E, Hutchison ER, Marosi K, Comotto J, Mustapic M, Nigam SM, et al. Extracellular vesicle-associated $A \beta$ mediates trans-neuronal bioenergetic and Ca. NPJ Aging Mech Dis. 2016;2.

54. Joshi P, Benussi L, Furlan R, Ghidoni R, Verderio C. Extracellular vesicles in Alzheimer's disease: friends or foes? Focus on aß-vesicle interaction. Int J Mol Sci. 2015;16(3):4800-13.

55. Yuyama K, Sun $H$, Mitsutake $S$, Igarashi $Y$. Sphingolipid-modulated exosome secretion promotes clearance of amyloid- $\beta$ by microglia. J Biol Chem. 2012; 287(14):10977-89.

56. Katsuda T, Tsuchiya R, Kosaka N, Yoshioka Y, Takagaki K, Oki K, et al. Human adipose tissue-derived mesenchymal stem cells secrete functional neprilysin-bound exosomes. Sci Rep. 2013;3:1197. 
57. Maas SLN, Breakefield XO, Weaver AM. Extracellular vesicles: unique intercellular delivery vehicles. Trends Cell Biol. 2017;27(3):172-88.

58. Kim S, Sato Y, Mohan PS, Peterhoff C, Pensalfini A, Rigoglioso A, et al. Evidence that the rab5 effector APPL1 mediates APP-BCTF-induced dysfunction of endosomes in Down syndrome and Alzheimer's disease. Mol Psychiatry. 2016;21(5):707-16.

59. Hébert SS, Horré K, Nicolaï L, Papadopoulou AS, Mandemakers W, Silahtaroglu AN, et al. Loss of microRNA cluster miR-29a/b-1 in sporadic Alzheimer's disease correlates with increased BACE1/beta-secretase expression. Proc Natl Acad Sci U S A. 2008;105(17):6415-20.

60. Sun R, Liu Y, Lu M, Ding Q, Wang P, Zhang H, et al. ALIX increases protein content and protective function of iPSC-derived exosomes. J Mol Med (Berl). 2019;97(6):829-44.

61. Pera M, Larrea D, Guardia-Laguarta C, Montesinos J, Velasco KR, Agrawal RR, et al. Increased localization of APP-C99 in mitochondria-associated ER membranes causes mitochondrial dysfunction in Alzheimer disease. EMBO J. 2017;36(22):3356-71.

62. Murphy MP. Mitochondrial dysfunction indirectly elevates ROS production by the endoplasmic reticulum. Cell Metab. 2013;18(2):145-6.

63. Jeong SY, Seol DW. The role of mitochondria in apoptosis. BMB Rep. 2008; 41(1):11-22.

64. Yamasaki A, Tani K, Yamamoto A, Kitamura N, Komada M. The Ca2+ -binding protein ALG-2 is recruited to endoplasmic reticulum exit sites by Sec31A and stabilizes the localization of Sec31A. Mol Biol Cell. 2006;17(11): 4876-87.

65. Rider MA, Cheerathodi MR, Hurwitz SN, Nkosi D, Howell LA, Tremblay DC, et al. The interactome of EBV LMP1 evaluated by proximity-based BioID approach. Virology. 2018;516:55-70,

66. Young-Pearse TL, Bai J, Chang R, Zheng JB, LoTurco JJ, Selkoe DJ. A critical function for beta-amyloid precursor protein in neuronal migration revealed by in utero RNA interference. J Neurosci. 2007;27(52):14459-69.

67. Shipley MM, Mangold CA, Szpara ML. Differentiation of the SH-SY5Y human neuroblastoma cell line. J Vis Exp. 2016;108:53193.

68. Hurwitz SN, Meckes DG. An adaptable polyethylene glycol-based workflow for proteomic analysis of Extracellar vesicles. Methods Mol Biol. 2017;1660: 303-17.

69. Hurwitz SN, Cheerathodi MR, Nkosi D, York SB, Meckes DG. Tetraspanin CD63 bridges autophagic and endosomal processes to regulate exosomal secretion and intracellular signaling of Epstein-Barr virus LMP1. J Virol. 2017.

70. Rider MA, Hurwitz SN, Meckes DG. ExtraPEG: a polyethylene glycolbased method for enrichment of extracellular vesicles. Sci Rep. 2016;6: 23978.

71. Lötvall J, Hill AF, Hochberg F, Buzás El, Di Vizio D, Gardiner C, et al. Minimal experimental requirements for definition of extracellular vesicles and their functions: a position statement from the International Society for Extracellular Vesicles. J Extracell Vesicles. 2014;3:26913.

72. Witwer KW, Soekmadji C, Hill AF, Wauben MH, Buzás El, Di Vizio D, et al. Updating the MISEV minimal requirements for extracellular vesicle studies: building bridges to reproducibility. J Extracell Vesicles. 2017;6(1): 1396823.

73. Lässer C, Eldh M, Lötvall J. Isolation and characterization of RNA-containing exosomes. J Vis Exp. 2012;59:e3037.

74. Hurwitz SN, Conlon MM, Rider MA, Brownstein NC, Meckes DG Nanoparticle analysis sheds budding insights into genetic drivers of extracellular vesicle biogenesis. J Extracell Vesicles. 2016;5:31295.

75. Nazarenko I, Rupp AK, Altevogt P. Exosomes as a potential tool for a specific delivery of functional molecules. Methods Mol Biol. 2013;1049:495-511.

76. Ippolito DM, Eroglu C. Quantifying synapses: an immunocytochemistrybased assay to quantify synapse number. J Vis Exp. 2010;45.

77. Yan Y, Bejoy J, Xia J, Guan J, Zhou Y, Li Y. Neural patterning of human induced pluripotent stem cells in 3-D cultures for studying biomoleculedirected differential cellular responses. Acta Biomater. 2016;42:114-26.

78. Yan Y, Martin LM, Bosco DB, Bundy JL, Nowakowski RS, Sang QX, et al. Differential effects of acellular embryonic matrices on pluripotent stem cell expansion and neural differentiation. Biomaterials. 2015;73:231-42.

79. Bejoy J, Song L, Zhou Y, Li Y. Wnt/yes-associated protein interactions during neural tissue patterning of human induced pluripotent stem cells. Tissue Eng Part A. 2018;24(7-8):546-58.

80. Yan Y, Song L, Madinya J, Ma T, Li Y. Derivation of cortical spheroids from human induced pluripotent stem cells in a suspension bioreactor. Tissue Eng Part A. 2018;24(5-6):418-31.
81. Bejoy J, Song L, Wang Z, Sang QX, Zhou Y, Li Y. Neuroprotective activities of heparin, Heparinase III, and hyaluronic acid on the a. ACS Biomater Sci Eng. 2018;4(8):2922-33

82. Marzano M, Bejoy J, Cheerathodi MR, Sun L, York SB, Zhao J, et al. Differential Effects of Extracellular Vesicles of Lineage-Specific Human Pluripotent Stem Cells on the Cellular Behaviors of Isogenic Cortical Spheroids. Cells. 2019;8(9).

\section{Publisher's Note}

Springer Nature remains neutral with regard to jurisdictional claims in published maps and institutional affiliations.
Ready to submit your research? Choose BMC and benefit from:

- fast, convenient online submission

- thorough peer review by experienced researchers in your field

- rapid publication on acceptance

- support for research data, including large and complex data types

- gold Open Access which fosters wider collaboration and increased citations

- maximum visibility for your research: over $100 \mathrm{M}$ website views per year

At BMC, research is always in progress.

Learn more biomedcentral.com/submissions 